\title{
Computational models for active matter
}

\author{
M. Reza Shaebani ${ }^{1}$, Adam Wysocki ${ }^{1}$, Roland G. Winkler ${ }^{2}$, Gerhard Gompper ${ }^{2}$ \& Heiko Rieger ${ }^{1}$ \\ ${ }^{1}$ Department of Theoretical Physics \& Center for Biophysics, Saarland University, 66123 Saarbrücken, Germany \\ ${ }^{2}$ Theoretical Soft Matter and Biophysics, Institute of Complex Systems \& Institute for Advanced Simulation, \\ Forschungszentrum Jülich, 52425 Jülich, Germany
}

\begin{abstract}
A variety of computational models have been developed to describe active matter at different length and time scales. The diversity of the methods and the challenges in modeling active matterranging from molecular motors and cytoskeletal filaments over artificial and biological swimmers on microscopic to groups of animals on macroscopic scales-mainly originate from their out-ofequilibrium character, multiscale nature, nonlinearity, and multibody interactions. In the present review, various modeling approaches and numerical techniques are addressed, compared, and differentiated to illuminate the innovations and current challenges in understanding active matter. The complexity increases from minimal microscopic models of dry active matter toward microscopic models of active matter in fluids. Complementary, coarse-grained descriptions and continuum models are elucidated. Microscopic details are often relevant and strongly affect collective behaviors, which implies that the selection of a proper level of modeling is a delicate choice, with simple models emphasizing universal properties and detailed models capturing specific features. Finally, current approaches to further advance the existing models and techniques to cope with real-world applications, such as complex media and biological environments, are discussed.
\end{abstract}

Active matter consists of particles, agents, or constituents that consume energy and convert it into directed motion, generate forces and shape deformations, or even proliferate and annihilate. Active matter systems are therefore manifestly out of equilibrium, and the local energy consumption discriminates them from other out-of-equilibrium systems, in which energy is injected, e.g., via the boundaries like in shear flow. Some of the basic features of active matter are depicted in Box 1a. New models, methods, and computational techniques have been developed in the last two decades to understand and unravel the unique physical principles governing active matter [1-5]. Of particular interest are novel many-particle effects or collective phenomena, like motility-induced phase separation, spontaneous rotational symmetry breaking in two dimensions, pattern formation, and self-organization. Living systems are paradigmatic examples of active matter, where active units reproduce, adapt, and dynamically respond to environmental changes. The huge diversity of active agents and their wide range of behaviors are major challenges in developing a comprehensive theoretical description of living matter. A large variety of numerical methods with different levels of resolution, ranging from micro- to macroscale, have been developed and employed to model living and artificial active matter [1-5], as summarized in Fig. 1. The goal of this review is to summarize, compare, and differentiate between currently available models and to elucidate existing challenges in computational modeling of active matter.

We first consider dry active matter, i.e., systems where hydrodynamic interactions are absent and momentum is not conserved. Next, approaches to model hydrodynamics of active suspensions are discussed. Here, the dynamics of the solvent is incorporated in the model, ensuring local momentum conservation. Moreover, we present an overview of the continuum models employed for active fluids, and elucidate the practical relevance of the numerical approaches. The degree of coarse-graining determines the details of real systems that can be captured, which becomes most evident in the modeling of cells, tissues, and animal groups. Finally, we address the open challenges that modeling active matter is currently facing, and present an outlook on model developments toward real-world applications.

\section{DRY ACTIVE MATTER}

Dry active systems are characterized by the absence of momentum conservation. This can be due to contact with a momentum-absorbing medium, as in gliding of bacteria or vibration of granular beads on frictional surfaces. The omission of momentum conservation may also originate from the minor relevance of hydrodynamic interactions in systems where other effects, such as fluctuations, volume exclusion, and short-range or metric-free interactions, dominate. Relevant examples include animal flocks and swimming of dense bacterial collections. Specifically, we discuss two paradigmatic minimal models of dry active matter and their variants and extensions: active Brownian particles and Vicsek-type models with alignment interactions. In addition, we briefly address continuum-modeling approaches. Neglecting birth, division, and death processes, we here only consider systems in which the number of particles is conserved.

\section{Active Brownian particles}

A rather generic model of an active agent is the active Brownian particle (ABP), a self-propelled spherical particle whose dynamics is described by the overdamped Langevin equations

$$
\begin{aligned}
\frac{\mathrm{d} \boldsymbol{r}(t)}{\mathrm{d} t} & =\frac{D}{k_{B} T}\left[-\nabla U(\boldsymbol{r})+\boldsymbol{F}_{\text {active }}(\boldsymbol{\theta})+\boldsymbol{\xi}(t)\right], \\
\frac{\mathrm{d} \boldsymbol{\theta}(t)}{\mathrm{d} t} & =-\frac{D_{r}}{k_{B} T} \boldsymbol{\xi}_{r}(t),
\end{aligned}
$$

for the position $\boldsymbol{r}(t)$ and the orientation vector $\boldsymbol{\theta}(t)$. D and $D_{r}$ are the translational and rotational diffusion coefficient, 
$\boldsymbol{\xi}$ and $\boldsymbol{\xi}_{r}$ uncorrelated random force and torque (white noise), $\boldsymbol{F}_{\text {active }}$ a self-propulsion force (usually along the current direction of motion, i.e., $\boldsymbol{F}_{\text {active }}=F_{\circ} \frac{\boldsymbol{\theta}}{|\boldsymbol{\theta}|}$ ), and $U(\boldsymbol{r})$ is either the interaction energy with other particles or an external potential. The overdamped limit is suitable for motion in low-Reynolds-number fluids as, e.g., in biological environments. In order to model experimental systems, the equations of motion 1 often have to be generalized and adapted to cope with specific applications. Some of the extensions of the ABP model are discussed in Box 1b [6-13]. Other approaches to simulate self-propelled particle systems include active lattice gas model and (kinetic) Monte Carlo approach [14-20].

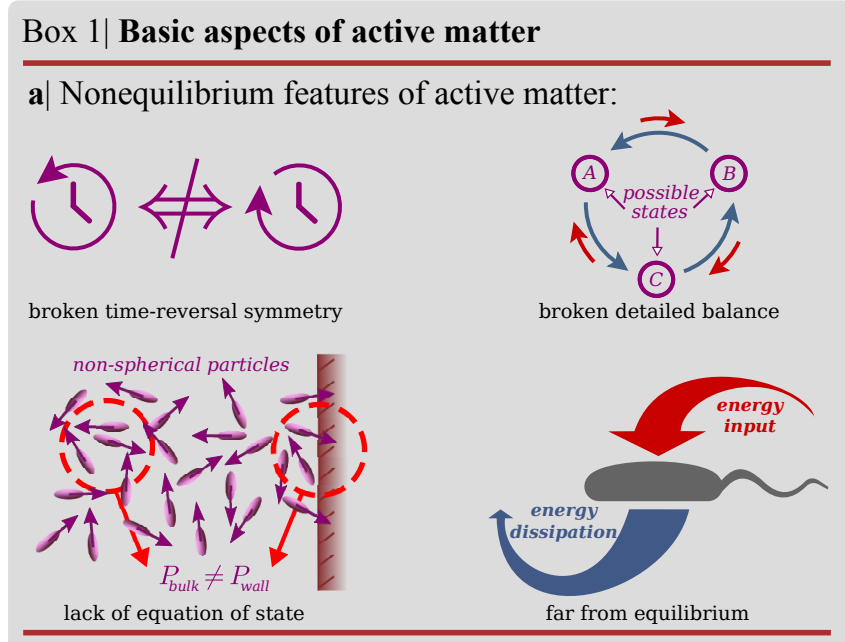

b) Shape and architecture: Extensions of the active Brownian sphere model include additional active torques ${ }^{7,8}$, asymmetric shapes, e.g. rods $^{9}$ and L-shaped microswimmers ${ }^{10}$, and assemblies of several active particles, e.g. in a polymerlike manner ${ }^{11-13}$. This leads to new emerging effects such as suppression of the motility-induced phase separation and appearance of large-scale coherent motion ${ }^{7}$, chiral motion patterns ${ }^{8}$, and an intimate coupling of activity and polymer conformations and dynamics ${ }^{11-13}$.

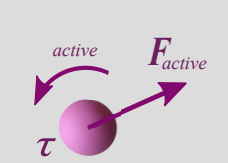

translational vs angular persistency

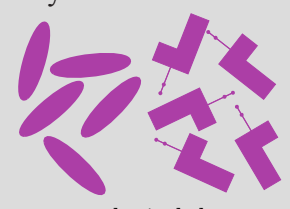

non-spherical shapes

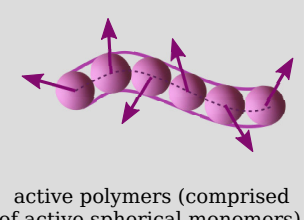

active polymers (comprised
Active particles, even with purely repulsive interactions, exhibit novel features, such as motility-induced phase separation (MIPS) [4, 5, 21-28], wall accumulation [29-31], capillary action in spite of wall-particle repulsion [32], and an active pressure (denoted as swim pressure) [31, 33-36]. Intuitively, the separation into dense and dilute regions of ABPs is explained by a positive feedback between blocking of persistent particle motion by steric interactions, and an enhanced probability of collisions with further particles at sufficiently large concentrations. The reduction in speed by collisions implies a local increase in density, which further increases the collision frequency in those regions. This mechanism eventually leads to particle accumulation and phase separation. During MIPS, the phase-separated domains grow self-similarly with time and their size is only limited by the system dimensions [23, 24]. Remarkably, in three dimensions, ABPs exhibit collective motion in the high-density phase-separated state without alignment rule [23]. Similarly, wall accumulation emerges by the (slow) orientational diffusion of adsorbed ABPs, which are only able to escape when their propulsion direction points away from the wall $[4,29]$. The strength of the effects depends on the rotational diffusion coefficient, the propulsion velocity, and the curvature of the surface. Simulation show that ABPs accumulate preferentially in regions of highest curvature [30, 37]. While non-equilibrium active systems usually lack a free energy and equations of state, spherical ABPs are a notable exception. Analytical considerations and simulations yield a pressure equation of state in this case [31, 33-36]; however, such equation does not exist for nonspherical, elongated ABPs [34].

\section{Active motion with alignment interactions}

Collective motion in various systems of living organisms (e.g., flocks of birds and animal herds) and ensembles of synthetic elongated active particles shares intriguing common features—such as swirling patterns and swarming-related to the alignment of motion with their neighbors [42].

Vicsek et al. proposed an agent-based minimal model for flocking, accounting for the interplay between fluctuations and simultaneous interactions of multiple agents [43]. Particles moving in a plane with constant velocity $v_{0}$ align with their neighbors by updating their direction of motion at each time step according to

$$
\boldsymbol{\theta}_{i}(t+1)=\left\langle\boldsymbol{\theta}_{i}(t)\right\rangle_{R}+\boldsymbol{\xi}_{i}(t),
$$

where $\left\langle\boldsymbol{\theta}_{i}(t)\right\rangle_{R}$ is the average orientation vector of particles located in a circle of radius $R$ surrounding particle $i$, and $\boldsymbol{\xi}_{i}(t)$ is a random vector with orientation obtained from a uniform distribution $[-\sigma \pi, \sigma \pi]$ along the current direction of motion. The strength of the noise is tuned by the parameter $\sigma \in[0,1]$. The new position of particle $i$ is then obtained as $\boldsymbol{x}_{i}(t+1)=\boldsymbol{x}_{i}(t)+v_{0} \hat{\boldsymbol{\theta}}_{i}$, where $\hat{\boldsymbol{\theta}}_{i}$ is the unit vector in the direction of $\boldsymbol{\theta}_{i}(t+1)$ and $v_{0}$ the velocity. A continuous transition from disordered to an ordered state occurs upon increasing the particle density or decreasing the noise strength $\sigma$. The global mean normalized velocity $m=\left|\sum_{i=1}^{N} \boldsymbol{v}_{\boldsymbol{i}}\right| /\left(N v_{0}\right)$ is an appropriate order parameter, characterizing the transition from random $(m=0)$ to coherent movement $(m=1)$. Despite of being a nonequilibrium phase transition, various notions of equilibrium statistical mechanics are borrowed here due to spontaneous symmetry breaking and emergence of well-defined macroscopic states.

According to numerous studies [42], the way in which noise and disorder are introduced into the system [44-46], boundary conditions (periodic vs reflecting, shape, etc.) [47], range [48] and type of interactions (including hard core, metric-free, repulsive or attractive) [49-52], and alignment rules (e.g., polar or bipolar) [53-55] influence the resulting patterns (leading to band formation $[52,56]$, rotating chains [57], marching groups, etc.) and the essential characteristics of collective motion such as the nature of the phase transition. For example, the order of the transition depends on: (i) whether the noise is intrinsic (perturbing the final orientation) or extrinsic (perturbing the individual 


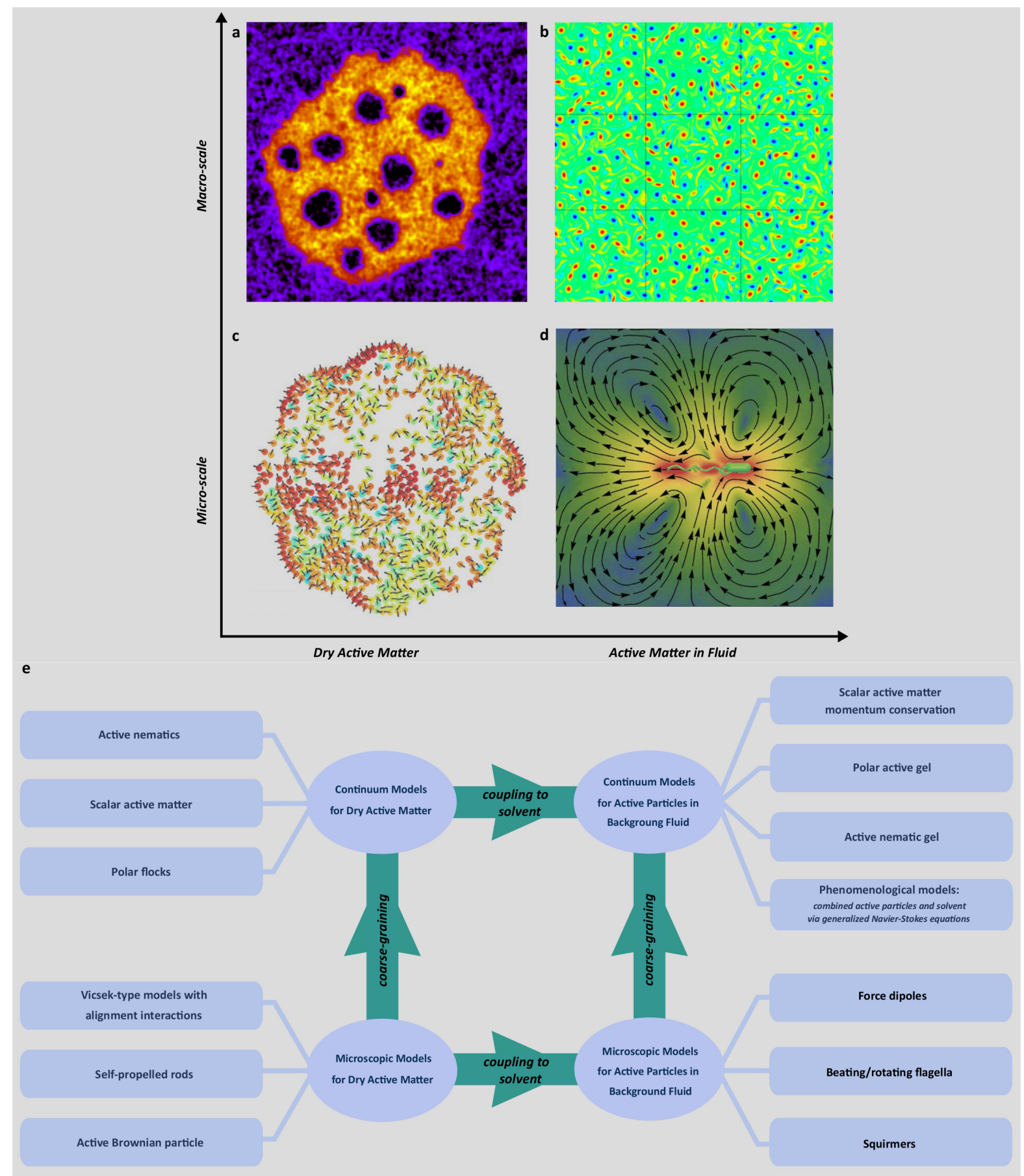

FIG. 1 | Modeling active matter. a-d| Typical numerical snapshots of active systems in (dry-wet, micro-macro) phase space. a| Bubbly phase separation in the density profile of a continuum dry active matter model. Boiling liquid and vapor phases are indicated with yellow and blue colors, respectively. Adapted from [38]. b| Vorticity field in the turbulent regime of a continuum active fluid model. Adapted from [39]. c| Ordering of vibrated polar disks in confinement. The color code denotes the relative alignment of dry active particle to the neighboring particles, ranging from blue (anti-parallel) to red (parallel). Adapted from [40]. d| Flow field generated by a single swimming bacterium. Adapted from [41]. e| Schematic drawing of the main computational models and methods for active matter. The arrows indicate the direction of generalization or increasing complexity in approaches, from microscopic to continuum models, and from dry active matter to active motion with coupling to a momentum-conserving solvent. 


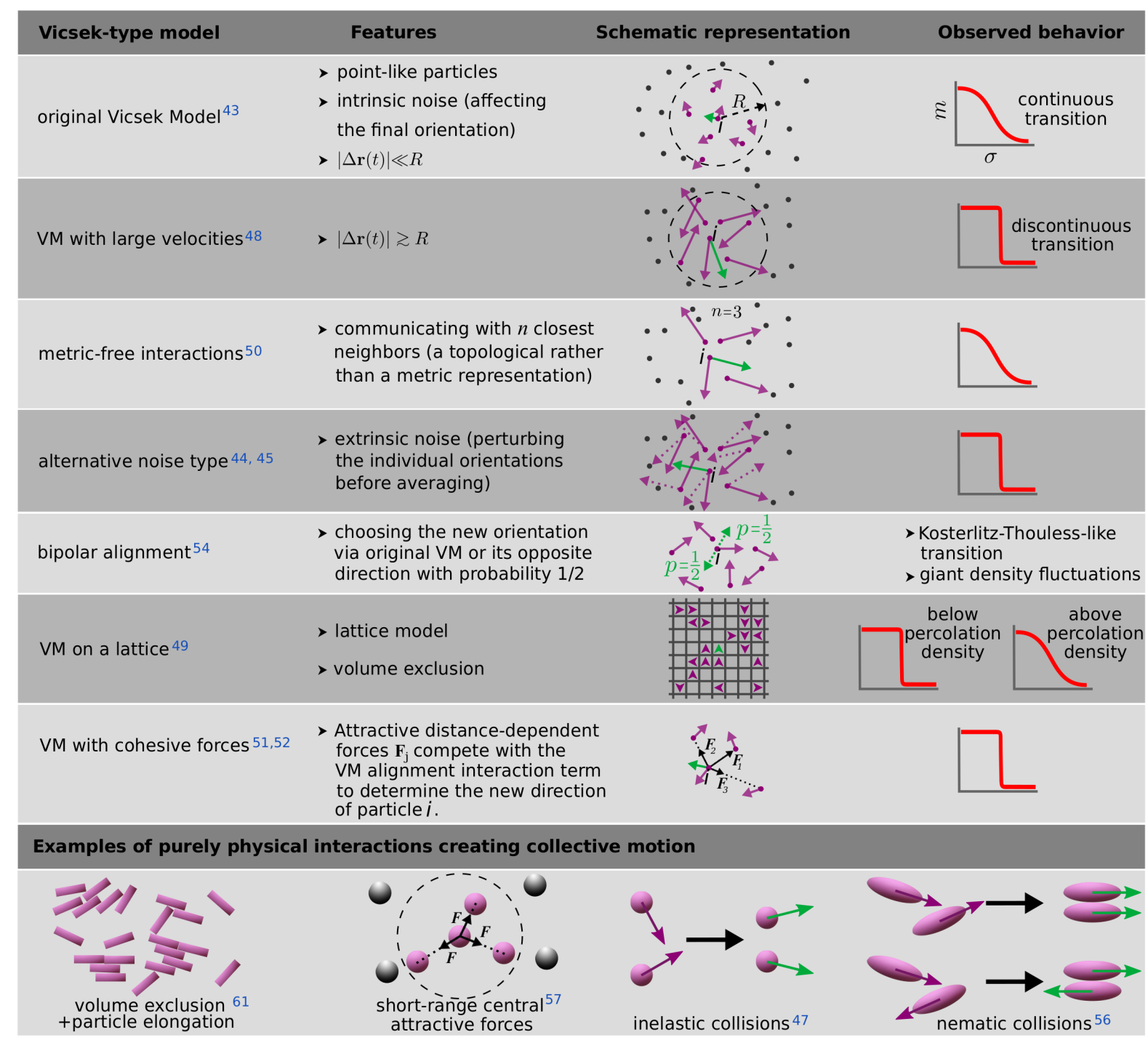

TABLE I | (top) A few variants of Vicsek model. The green vectors indicate the adapted orientation of the central particle $i$. The purple arrows are the directions that enter in the calculation for the new direction of particle $i . \sigma, m, R$, and $\Delta \boldsymbol{r}(t)$ represent, respectively, the noise strength, order parameter, radius of interaction, and particle displacement during one time step. (bottom) Examples of models exhibiting collective motion in active particle systems solely based on physical interactions without explicit alignment rules. 
orientations before averaging) [44, 45], (ii) whether interactions are metric (fixed range) or topological (fixed number of partners) [50], and (iii) the relative magnitude of the particle displacement $v_{0} \Delta t$ during one time step compared to the radius of interaction $R$ [48]. Recent studies indicate that the flocking transition in the Vicsek model is reminiscent of a liquid-gas transition, rather than an order-disorder transition, but with microphase separation in the coexistence region, where traveling ordered bands of finite width coexist with a disordered gas [58]. By contrast, the corresponding lattice model, called active Ising model, exhibits full phase separation [59]. In animal herds, agents sense the motion of their neighbors, resulting in polar alignment. However, an explicit (polar) alignment rule is not a necessary condition for coherent motion: the alignment can be induced implicitly through purely physical local interactions such as inelastic [47] or nematic collisions [56], short-range interactions $[57,60]$, or volume exclusion in combination with shape-induced (particle elongation) effects [61]. An important question is the minimal requirements for emerging collective motion by solely physical interactions. Table I lists some variants of the Vicsek model as well as a few systems which show collective motion without explicit alignment rules.

\section{Continuum models of dry active matter}

The large-scale behavior of many-particle systems can be captured by continuum models, which describe the evolution of continuous slow variables such as number density $n(\boldsymbol{r}, t)$ and velocity $\boldsymbol{v}(\boldsymbol{r}, t)$ fields. Such macroscopic approaches capture the major features of active matter, e.g., collective motion, by considering the conservation laws and broken continuous symmetries. Continuum theories can be constructed via coarse graining of a microscopic model, adopting symmetry arguments, or using out-of-equilibrium thermodynamics close to equilibrium. Representative continuum models of dry active matter, which conserve the number of particles while the momentum is not conserved, are provided in Table II.

Dry polar flocks - A continuum description of the Vicsek model, solely based on symmetry considerations, was first proposed by Toner and Tu [3, 62-65]. The theory describes active particles distinguishing front from rear and, therefore, are characterized by a polarization field $\boldsymbol{p}(\boldsymbol{r}, t)$ corresponding to the vectorial orientation of active particles and their direction of swimming. The conservation of the number density, $n(\boldsymbol{r}, t)$, results in a continuity equation including an active contribution $v_{0} n \boldsymbol{p}$ to the particle flux (see equation A1 in Table II). The simplest form of the dynamical equation for $\boldsymbol{p}$ assumes a relaxation process toward the minimum of an effective free energy $\mathscr{F}[n, \boldsymbol{p}]$ including, amongst others, terms responsible for the spontaneous polarization and the energetic cost due to elastic distortions of the orientational field. As shown in equation A2, activity enters solely via an advective term $\lambda(\boldsymbol{p} \cdot \boldsymbol{\nabla}) \boldsymbol{p}$, which accounts for the fact that distortions in $\boldsymbol{p}$ are advected by itself because $\boldsymbol{p}$ represents both the order parameter and the velocity. Flocks do not conserve momentum and the system lacks Galilean invariance, therefore, $\lambda \neq v_{0}$, meaning that density and polarization inhomogeneities advect at different velocities. The model exhibits giant number fluctuations and long-range order in $2 \mathrm{D}$ (forbidden in thermal equilibrium by the Mermin-Wagner theorem) $[3,62]$. By relaxing the constraint on numberdensity conservation, the model can be generalized and applied to systems involving birth-division-death processes [66] as, e.g., in dense bacterial colonies.

Dry active nematics - A system of apolar (or head-tail symmetric) active particles can exhibit a state with long-range directional order, however, with zero global drift velocity due to the nematic symmetry. Active nematics can be characterized by a symmetric second-rank tensor $Q(r, t)$ representing the local alignment of the neighboring particles (see equation A3). The overdamped dynamics of $\boldsymbol{Q}$ obeys equation A4, reducing to the free energy $\mathscr{F}[n, Q]$ consisting of entirely quasi-passive terms. The continuity equation A5 for the density $n(\boldsymbol{r}, t)$ contains an active current $\boldsymbol{J}_{\text {active }}=\zeta \boldsymbol{\nabla} \cdot \boldsymbol{Q}[67,68]$ originating from the force dipole of active particles, which accounts for a particle flux along or against the curvature $\boldsymbol{\nabla} \cdot \boldsymbol{Q}$, thus violating the time-reversal symmetry. Despite the apparent simplicity, active nematics exhibit interesting behavior, e.g., giant number fluctuations and self-propulsion of topological defects $[1,67,68]$. An extension to a more complex environment, e.g., viscoelasticity by polymers [69], implies additional effects, such as drag reduction, spontaneous flows by an antagonistic coupling between polymer and nematic orientations, and active turbulence in a sufficiently soft elastomeric solid [70].

Dry scalar active matter - Anisotropic interactions are responsible for orientational order-disorder transitions in active nematics or polar matter. In contrast, active particles with spherical symmetry (i.e., without alignment interactions) do not display a global directional order $(p=Q=0)$ and the only remaining slow variable is the scalar number density $n$. To explore the physics of motility-induced phase separation in scalar active matter, the passive Model B [71]- a field-theoretical model for diffusive phase separation of the number density (characterized by a conserved scalar order parameter $\phi$ ) - has been extended by an active chemical potential $\mu_{\text {active }}=\lambda(\nabla \phi)^{2}$ that breaks the timereversal symmetry at leading order in the density gradient expansion [38, 72-75]. To allow for circulating real-space particle currents in steady state, further terms that break the gradient structure of the active current have been added [38] (see equations A6 and A7). The resulting active model $\mathrm{B}+(\mathrm{AMB}+)$ displays complex dynamics such as microphase separation (bubbles of a finite length-scale) and reverse Ostwald process (increase of the number of small bubbles while bigger bubbles evaporate) [38].

\section{ACTIVE PARTICLES IN FLUIDS}

Hydrodynamic interactions are fundamental for active particles immersed in a fluid, and determine their behavior in various respects. On the one hand, they are an integral part of the propulsion system of most of the biological and various synthetic microswimmers-without hydrodynamic interactions, no propulsion [4]. On the other hand, hydrodynamics determines the behavior of microswimmers at walls, in channels, as well as their collective behavior. Microscale modeling approaches provide insight into the underlying physical mechanism from the level of individual microswimmers up to the emergent collective behaviors on large length scales. 
Dry active matter

\begin{tabular}{|c|c|c|c|}
\hline Contiuum model & Governing equations & & Parameters \\
\hline $\begin{array}{l}\text { Toner-Tu Model } \\
\text { (dry polar flocks) }\end{array}$ & $\begin{array}{l}\partial_{t} n+\nabla \cdot\left(v_{0} n \boldsymbol{p}+\boldsymbol{J}_{\text {passive }}\right)=0 \\
\partial_{t} \boldsymbol{p}+\lambda(\boldsymbol{p} \cdot \boldsymbol{\nabla}) \boldsymbol{p}+\cdots=-\Gamma_{r} \frac{\delta \mathcal{F}[n, \boldsymbol{p}]}{\delta \boldsymbol{p}}+\chi\end{array}$ & A1 & $\begin{array}{l}v_{0}: \text { propulsion speed } \\
\lambda: \text { coefficient of convective derivative } \\
J_{\text {passive }}: \text { particle flux } \\
\chi: \text { random noise vector } \\
\mathcal{F}[n, p]: \text { free energy functional }\end{array}$ \\
\hline $\begin{array}{l}\text { Models of dry } 67,68 \\
\text { active nematics }\end{array}$ & $\begin{array}{l}\mathbf{Q}(\mathbf{r}, t)=S\left(p \otimes p-\frac{1}{d} \mathbf{I}\right) \\
\partial_{t} \mathbf{Q}=-\Gamma_{r} \frac{\delta \mathcal{F}[n, \mathbf{Q}]}{\delta \mathbf{Q}}+\Lambda \\
\partial_{t} n+\nabla \cdot\left(\zeta \nabla \cdot \mathbf{Q}+J_{\text {passive }}\right)=0\end{array}$ & $\begin{array}{l}\text { A4 } \\
\text { A5 }\end{array}$ & $\begin{array}{l}S: \text { magnitude of the order parameter } \\
\mathcal{F}[n, \mathrm{Q}]: \text { free energy functional } \\
\Lambda: \text { white noise tensor } \\
\zeta: \text { coefficient of active current }\end{array}$ \\
\hline $\begin{array}{l}\text { Scalar active }{ }^{72-75} \\
\text { model } \mathrm{B}+\end{array}$ & $\begin{array}{l}\phi=\left(2 n-n_{H}-n_{L}\right) /\left(n_{H}-n_{L}\right) \\
\partial_{t} \phi+\nabla \cdot\left(-\lambda \nabla\left((\nabla \phi)^{2}\right)+\xi\left(\nabla^{2} \phi\right) \nabla \phi+J_{\text {passive }}+\chi\right)=0\end{array}$ & A6 & $\begin{array}{l}n_{H}, n_{L}: \text { densities of high and low density } \\
\text { coexisting phases } \\
\lambda, \xi \text { terms break time-reversal symmetry. }\end{array}$ \\
\hline
\end{tabular}

\section{Active particles in fluid}

\begin{tabular}{|c|c|c|c|}
\hline \multirow{3}{*}{$\begin{array}{l}\text { Contiuum model } \\
\\
\text { Polar active }{ }^{1-3,} 178-183 \\
\text { gel models }\end{array}$} & Governing equations & \multirow{2}{*}{\multicolumn{2}{|c|}{$\begin{array}{l}\quad \text { Parameters } \\
\mathbf{f}: \text { force density } \\
\boldsymbol{\sigma}=\boldsymbol{\sigma}^{a}+\boldsymbol{\sigma}^{d}+\boldsymbol{\sigma}^{r}: \text { stress tensor } \\
\boldsymbol{\sigma}^{a}: \text { ative stress } \\
\boldsymbol{\sigma}^{d}=2 \eta \mathbf{E}: \text { dissipative stress } \\
\mathbf{E}=\frac{1}{2}\left(\nabla v+(\nabla v)^{T}\right): \text { rate of strain tensor } \\
\boldsymbol{\sigma}^{r}: \text { reversible stress due to free energy functional }\end{array}$}} \\
\hline & $\begin{array}{l}\text { incompressible Navier-Stokes equations: } \\
\left\{\begin{array}{l}\rho\left(\partial_{t}+\boldsymbol{v} \cdot \nabla\right) v=-\nabla P+\nabla \cdot \boldsymbol{\sigma}+\mathbf{f} \\
\nabla \cdot \boldsymbol{v}=0\end{array}\right. \\
\text { Stokes equation: } \\
\nabla P-\nabla \cdot \boldsymbol{\sigma}-\mathbf{f}=0\end{array}$ & & \\
\hline & $\begin{array}{l}D_{t} \boldsymbol{p}+\lambda_{1}(\boldsymbol{p} \cdot \boldsymbol{\nabla}) \boldsymbol{p}+\cdots=\lambda \mathbf{E} \cdot \boldsymbol{p}-\Gamma_{r} \frac{\delta \mathcal{F}}{\delta \boldsymbol{p}} \\
\partial_{t} n+\nabla \cdot[(\boldsymbol{p}+\boldsymbol{v}) n]=0\end{array}$ & B3 & $\begin{array}{l}D_{t}: \text { convected corotational time derivative } \\
\lambda_{1}: \text { strength of advection by polarization } \\
\lambda: \text { flow alignment coefficients } \\
\mathcal{F}[p] \text { : free energy functional containing Frank } \\
\text { elastic terms and terms controlling the } \\
\quad \text { order-disorder transition }\end{array}$ \\
\hline $\begin{array}{l}1-3,90 \\
\text { Active nematic } \\
\text { gel models }\end{array}$ & $\left(\partial_{t}+v \cdot \nabla\right) \mathbf{Q}=\mathbf{S}(\mathbf{E}, \Omega)+\Gamma \mathbf{H}$ & B5 & $\begin{array}{l}\mathbf{H} \text { : variational derivative of the free energy } \\
\Omega \text { : vorticity tensor } \\
\text { S describes the competition between rotation } \\
\text { and flow alignment. }\end{array}$ \\
\hline $\begin{array}{l}\text { Generalized }^{183,188-193} \\
\text { Navier-Stokes } \\
\text { models }\end{array}$ & $\begin{aligned} \mathbf{f} & =\frac{\delta F[v]}{\delta \boldsymbol{v}} \\
\boldsymbol{\sigma}^{a} & =-\zeta\left[\boldsymbol{v} \otimes \boldsymbol{v}-\frac{v^{2}}{d} \mathbf{I}\right] \\
\boldsymbol{\sigma}^{d} & =2\left[\Gamma_{0}-\Gamma_{2} \nabla^{2}+\Gamma_{4}\left(\nabla^{2}\right)^{2}\right] \mathbf{E}\end{aligned}$ & B7 & $\begin{array}{l}F[v] \text { : biquadratic Landau-like free energy } \\
\zeta \text { :activity parameter } \\
\boldsymbol{\sigma}^{d} \text { is a generalized dissipative stress. }\end{array}$ \\
\hline $\begin{array}{l}\text { Scalar active } 194,195 \\
\text { model H }\end{array}$ & $\begin{array}{l}\partial_{t} \phi+v \cdot \nabla \phi=\Gamma_{t} \nabla^{2} \mu \\
\mu=a \phi+b \phi^{3}-\kappa \nabla^{2} \phi+\lambda(\nabla \phi)^{2} \\
\boldsymbol{\sigma}=-\hat{\kappa}\left[(\nabla \phi) \otimes(\nabla \phi)-\frac{(\nabla \phi)^{2}}{d} \mathbf{I}\right]\end{array}$ & $\begin{array}{l}\text { B9 } \\
\text { B10 } \\
\text { B11 }\end{array}$ & $\begin{array}{l}\phi: \text { scalar order parameter } \\
\mu: \text { chemical potential } \\
\lambda(\nabla \phi)^{2}: \text { active contribution to } \mu \\
\hat{\kappa}=\kappa+\zeta \neq \kappa\end{array}$ \\
\hline
\end{tabular}

TABLE II | The governing equations of continuum models for dry active matter (top) and active particles in fluids (bottom). $P$ denotes pressure, $n, \rho$ number and mass density, $\boldsymbol{v}$ velocity, $\boldsymbol{Q}(\boldsymbol{r}, t)$ nematic alignment tensor, $\boldsymbol{p}$ polarization or nematic director, $\mathbf{I}$ identity tensor, $T$ temperature, $\Gamma_{t}, \Gamma_{r}$ translational and rotational mobility, and $\eta$ viscosity.

\section{Universal features}

The hydrodynamics of simple Newtonian fluids is governed by the Navier-Stokes equation, see equation $B 1$ in Table II. In the limit of low Reynolds numbers,

$$
R e=\rho v_{0} L / \eta \ll 1
$$

(with $L, v_{0}$, and $t_{0}=L / v_{0}$ being a characteristic length, swim velocity, and time), this equation reduces to the Stokes equation, where inertial terms are negligible (equation B2 in Table II). Characteristic values for microswimmers are body lengths $L \sim O(\mu \mathrm{m})$ and swimming velocities $v_{0} \sim O(\mu \mathrm{m} / \mathrm{s})$, hence, $R e \lesssim 10^{-3}$ for water. In this limit, hydrodynamics becomes time independent and the dynamics is reversible. This has the important consequence that a microswimmer with a time reversible stroke cannot propel (scallop theorem), as first pointed out in a seminal paper by Purcell [76].

The solution of the Stokes equation is determined by the Oseen tensor

$$
H_{\alpha \beta}(r)=\frac{1}{8 \pi \eta r}\left[\delta_{\alpha, \beta}+r_{\alpha} r_{\beta} / r^{2}\right], \quad(r=|r|)
$$

the Greens function of the Stokes equation. The flow field obtained from $\mathbf{H}$ for a point force is typically denoted as Stokeslet (cf. Box 2). It is important to note that the Stokeslet does not describe an autonomous microswimmer, because the swimmer must be force- and torque-free. Instead, a swimmer usually consists of a motor, propelling the fluid, and a cargo, being pushed or dragged forward. Approximating these two components by point forces with opposite directions $\hat{\boldsymbol{e}}$ and $-\hat{\boldsymbol{e}}$, and equal magnitude $f_{0}$, yields the dipole swimmer with the flow field of equation $\mathrm{C} 1$, where $P=f_{0} L$ is the dipole strength (cf. Box 2). The sign of $P$ distinguishes a pusher $(P>0$, motor in the back) from a puller $(P<0$, motor in front), with equal flow lines but opposite flow directions (cf. Box 2) [4]. The flow field of a microswimmer is typically more complex and comprises higher order multipoles [77, 78]. However, in the far-field (far away from the swimmer compared to its own size) the dipole contribution $\mathrm{C} 1$ dominates. 
a Far-field multipole expansion: The flow field of a microswimmer is expanded in terms of multipoles- force dipole (FD), source dipole (SD), force quadrupole (FQ), etc. $4,77: \boldsymbol{u}(\boldsymbol{r})=\boldsymbol{u}_{\mathrm{FD}}(\boldsymbol{r})+\boldsymbol{u}_{\mathrm{SD}}(\boldsymbol{r})+\boldsymbol{u}_{\mathrm{FQ}}(\boldsymbol{r})+\cdots$

The far-field is determined by the force dipole $\boldsymbol{u}_{\mathrm{FD}}(\boldsymbol{r})$ $(P \neq 0)$ or the source dipole $\boldsymbol{u}_{\mathrm{SD}}(\boldsymbol{r})(P=0)$ :

$\begin{array}{ll}\boldsymbol{u}_{\mathrm{FD}}(\boldsymbol{r})=\frac{P \boldsymbol{r}}{8 \pi \eta \mathrm{r}^{3}}\left(-1+3 \frac{(\boldsymbol{r} \cdot \hat{\boldsymbol{e}})^{2}}{\mathrm{r}^{2}}\right), & \mathrm{C} 1 \\ \boldsymbol{u}_{\mathrm{SD}}(\boldsymbol{r})=\frac{\kappa_{\mathrm{SD}}}{r^{3}}\left(\hat{\boldsymbol{e}}-\frac{3 z \boldsymbol{r}}{r^{2}}\right), & \mathrm{C} 2 \\ \text { with } \hat{\boldsymbol{e}} \text { being the propulsion direction. }\end{array}$
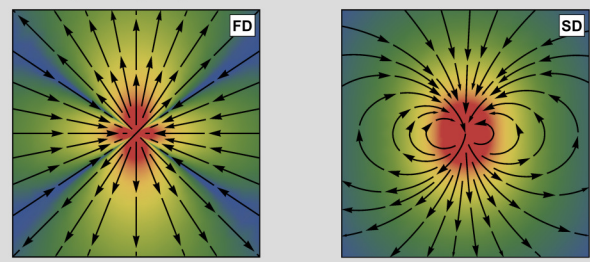

b| Coarse-grained microswimmer: Details of flow fields can be captured by the squirmer model of a microswimmer, a spheroidal solid body with a prescribed surface slip velocity.

For a sphere, the surface velocity is ${ }^{4}$

$\boldsymbol{u}_{\mathrm{sq}}=B_{1}(\sin \vartheta+\beta \sin \vartheta \cos \vartheta) \hat{\mathrm{e}}_{\vartheta}$,

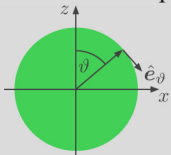

where $B_{1}=2 v_{0} / 3$ ( $v_{0}$ : swim velocity) and $\beta$ is the stresslet strength $(\beta<0$ pusher, $\beta>0$ puller). The figure shows a spheroidal puller with $\beta=3$ in the (left) laboratory frame and (right) body-fixed frame $e^{129}$
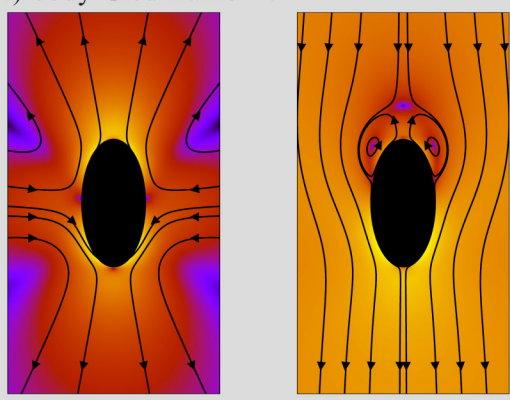

c| Explicit propulsion mechanism:

(i) Resistive force theory $y^{137}$ - Hydrodynamic interactions included by different friction coefficients parallel $(\|)$ and perpendicular $(\perp)$ to a rodlike segment of a flagellum

$\boldsymbol{F}=\zeta_{\|} \boldsymbol{v}_{\|}+\zeta_{\perp} \boldsymbol{v}_{\perp}$

The figure shows the time sequence of sperm flagellum beat from experiment (red) and resistive force theory (blue). Time increases from light to dark colors ${ }^{138}$.

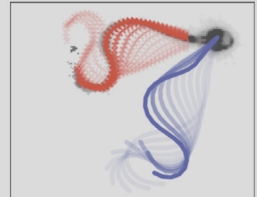

(ii) Explicit modeling of microswimmer and fluid- The complex flow pattern, including the near-field, are resolved by microscopic models, as illustrated for a swimming E. coli ${ }^{40}$ (left panel). Coupling with walls leads to a particular alignment of the swimmer axis and swimming direction as illustrated by trajectory of the sperm head ${ }^{113}$ (right panel).

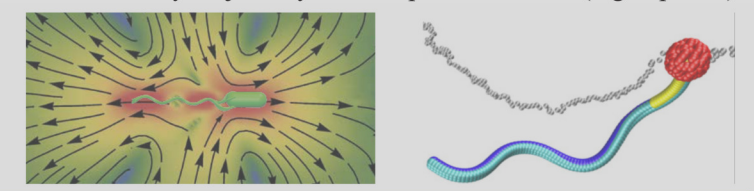

d| Swimming near surfaces: The hydrodynamic interactions of a microswimmer with a wall can be described by its interaction with an image (see left figure). The flow field for a no-slip boundary condition is ${ }^{4,78}$

$$
v_{w}=-\frac{P}{48 \pi \eta z_{0}^{2}}\left(1-3\left(\hat{\boldsymbol{e}} \cdot \hat{\boldsymbol{e}}_{z}\right)^{2}\right) \text {. }
$$

This leads to surface trapping and, e.g., the counterrotation of cell body and flagellum bundle to circular trajectories ${ }^{40}$, as shown in the right figure.
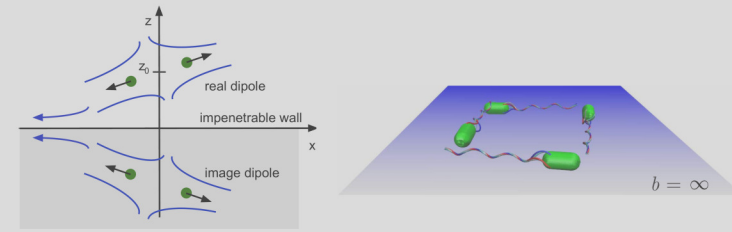

The dipole flow field has important consequences for the interaction of swimmers with walls $[4,77,79-81]$ and other swimmers. Here, inflow/outflow generates an effective attraction/repulsion. Together with the flow-induced torque, aligning pushers with a wall, pushers are attracted at walls. It should be noticed that flow-induced interactions add to an effective attraction emerging by propulsion, slow reorientation, and steric interactions, which also exists for dry active matter, and is thus independent of pusher- or pullertype swimming [29, 82-84]. In the latter case, the basic mechanism consists of arrival at the wall with the propulsion direction toward the wall, a slow reorientation determined 
by the rotational diffusion, during which the swimmer stays at the wall, and finally departure when the orientation points away from the wall. Hydrodynamics increases the wall detention time [85].

The hydrodynamic interactions also play an important role in the nematic arrangement of elongated or rodlike microswimmers $[86,87]$. When the aspect ratio exceeds about five, the nematic phase of passive rods is stable for sufficiently high volume fractions. Such an arrangement of microswimmers is sensitive to slight perturbations, e.g., by a sinusoidal reorientation wave. The flow field of pushers (pullers) at the nodes of this wave enhances (reduces) the perturbation, and thus destabilizes (stabilizes) the nematic phase. This hydrodynamic instability of the nematic phase of extensile active systems lies at the heart of the intriguing dynamics of active nematics [88-91].

Hydrodynamics can also lead to high-speed and longrange communication between freely swimming cells. For example, studies of the protist Spirostomum ambiguous suggest that long-ranged vortex flows can be generated by fast cell contraction, which, in turn, trigger contraction of neighboring cells and, hence, promote collective behavior [92].

\section{Swimming in viscoelastic fluids}

Microorganisms often move in complex environments, which are rather viscoelastic than Newtonian. Such environments break the time-reversal symmetry of Newtonian fluids, and allow for self-propulsion even for a time-symmetric internal motion, seemingly violating the scallop theorem [93]. Viscoelastic fluids are usually polymer solutions with a wide spectrum of properties, ranging from shear thinning to viscoelastic (characterized by storage and loss modulus), and, hence, can affect the swimming behavior of individual microswimmers [94, 95] as well as their collective properties [96]. Theoretical studies reveal both reduced [97, 98] and enhanced $[99,100]$ swim speeds, with a transition from slow small-amplitude swimming to fast large-amplitude locomotion [99]. Viscoelastic [99, 101] and shear thinning [102] effects are typically small. However, some unexpected phenomena can appear, which are related to the complex nature of polymer solutions. Microstructured fluids will generically phase-separate near surfaces, which can lead to the presence of low-viscosity fluid layers. Models show that this promotes slip and reduces viscous friction near the surface of the swimmer, which may increase swim speeds by orders of magnitude [100]. Experiments of E. coli in concentrated polymer solutions indicate that peculiarities of flagellated locomotion are indeed due to the fast-rotating flagellum, giving rise to a lower local viscosity in its vicinity [103]. A detailed modeling and simulation of bacteria in dense polymer solutions, with explicit polymers, reaches similar conclusions, specifically a depletion of polymers at the flagellum is obtained [104]. An increased swim speed with increasing polymer density is predicted, due to a nonuniform distribution of polymers in the vicinity of the bacterium, leading to an apparent slip, in combination with the chirality of the bacterial flagellum [104].

Hydrodynamics: mesoscale simulation techniques

Various hydrodynamic simulation approaches have been developed, which facilitate the study of mesoscopic activematter agents. Prominent mesoscale simulation approaches are the Lattice Boltzmann (LB) method [105, 106], Dissipative Particle Dynamics (DPD) [107], and the Multiparticle Collision Dynamics (MPC) approach [108, 109]. All these approaches are essentially alternative ways of solving the Navier-Stokes equation and its generalizations. The LB method yields an approximate solution of the Boltzmann equation, i.e., a single particle phase-space distribution function. An advantage of LB is that thermal fluctuations can be turned on and off as desired [106]. DPD and MPC are particle-based approaches, where the fluid is represented by point particles. The DPD dynamics proceeds analogous to traditional molecular dynamics (MD) simulations, however, with pairwise momentum-conserving stochastic and friction forces. By specific pairwise DPD particle interactions, compressibility of the fluid can be controlled. MPC consists of alternating streaming and collision steps, with a ballistic streaming motion and local momentum-conserving stochastic interactions (collisions), e.g., by rotation of relative velocities [109]. By the point-particle nature of the MPC particles, no fluid-induced depletion occurs, and a rather continuous representation of the fluid is obtained. Other simulation approaches implicitly take hydrodynamic interactions into account via a hydrodynamic tensor, e.g., Oseen tensor (equation 4) [4] or moment expansion [77, 110, 111] and mobilities [112].

Coupling of a microswimmer with the fluid can be achieved in various ways, depending on the nature of the microswimmer model and the desired extent of coarse-graining. Rather detailed models of, e.g., sperm and E. coli cells applying no-slip boundary conditions on the flagella and cell body, combined with a momentum conserving propulsion mechanism, e.g., rotation of a flagellum in case of a bacteria combined with counter-rotation of the cell body, leads to swimming motion. This applies to microswimmers in an explicit [41, 113] and implicit [114-116] solvent.

Squirmers are a coarse-grained representation of microswimmers, modeled as a colloid with prescribed fluid velocity at its surface (slip velocity $\boldsymbol{v}_{\text {sq }}$ ) [117-120]. This approach was originally designed for ciliated microswimmers, such as Paramecia [120]. Nowadays, it is considered as a generic model for a broad class of microswimmers, ranging from diffusiophoretic particles [5] to biological cells, and has been applied to study collective effects in bulk [119, 121126], at surfaces $[110,121,127,128]$, and in narrow slits $[129,130]$. Typically, the slip velocity of a sphere is approximated by equation C3 of Box 2 [118, 120, 129]. Extension to prolate spheroidal microswimmers have been proposed $[127,131,132]$. The squirmer model has been applied in combination with the boundary element method [119], the LB approach [121, 126, 128], and the MPC [130, 132] representation of the fluid.

A further level of coarse-graining is obtained by taking only far-field hydrodynamics into account, and representing a microswimmer as a force dipole (see above), where one particle is moving in the direction of the applied force and the total momentum is conserved by imposing the opposite force on the fluid [133]. Extensions to dumbbell-type swimmers [134] or even more complex spherical [135, 136] and rodlike $[83,135]$ structures have been proposed. Such an approach allows for the study of a large number of mi- 
croswimmers with a minimal numerical effort. However, the near-field is not adequately accounted for [135], which becomes relevant for the swimming behavior near a surface, in thin slits, or even the collective behavior in dense systems.

\section{Biological swimmers}

Cell motility is a major achievement of biological evolution and is essential for a wide spectrum of cellular activities, such as search for food, reproduction, or escape from predators [4]. The spectrum of microswimmers is wide, ranging from bacteria, e.g., Escherichia coli, protozoa, e.g., dinoflagellates, algae, e.g., Chlamydomonas reinhardtii, to spermatozoa. Unravelling the underlying propulsion mechanisms is essential for the understanding of their behavior, possible utilization of microswimmers in medicine, ecology, and technical applications, or for biomimetics by transferring biological concepts into synthetic swimmers. Biological microswimmers, both prokaryotes and eukaryotes, exploit flagella for propulsion, although the structure of their flagella differs $[4,80,137]$. Bacteria typically use one or several rotating helical flagella for locomotion, whereas a flagellum (or cilium) of an eukaryote beats in a wave-like fashion [138]. Explicit modeling of these microswimmers requires to account for three components: the cell body, the flagellum or several flagella, and the embedding fluid (see above). Typically, such cells are considered as neutrally buoyant objects, with spherical, spheroidal, or cylindrical cell body and (an) attached flagellum/flagella. Both parts are either considered as solid bodies [116, 137, 139, 140] or are composed of linked discrete points in a crane-like fashion for eukaryotic [113, 141] or bacteria [41, 114, 142-145] flagella. For the latter, traditional polymer models are employed, or a flagellum is described by the helical wormlike chain model [4]. A bacterium is propelled by independent rotation of flagella via an applied torque. Assigning the opposite forces and torques to the cell body ensures a force- and torque-free swimming [4]. In any case, propulsion is due to frictional anisotropy of the thin flagellum [4, 80, 140].

Simulation studies emphasize the importance of hydrodynamic interactions for, e.g., the synchronization of bacteria flagella in the bundling process, e.g., for E. coli [142, 145] or the beating of Chlamydomonas flagella. Moreover, they illustrate the complexity of the hydrodynamic flow field adjacent to a cell, which is important for cell-cell scattering/interaction processes [41]. Interactions of microswimmers with surfaces are fundamental in many biological processes, e.g., biofilm formation and egg fertilization. As pointed out above (see Box 2), surface hydrodynamic interactions determine the swimmer orientation [77]. In the far-field approximation, E. coli and sperm are pushers and orient preferentially parallel to a surface, whereas Chlamydomonas is a puller and correspondingly aligns perpendicular to surfaces. For bacteria, the rotation of the helical bundle and the counter-rotation of the cell body lead to circular trajectories with a handedness and circle radius depending on the surface-slip length [146, 147]—clockwise trajectories follow for no-slip [148] and counterclockwise trajectories for perfect-slip boundary conditions [149]. Simulations show that a cell is sensitive to nanoscale changes in the surface slip length, being itself significantly larger [146].

Bacterial suspension show an intriguing chaotic state of collective motion called active turbulence [150]. Simulation studies of the collective behavior of spherical squirmers show cluster formation in thin films with no-slip boundary conditions (quasi-2D) [130]. However, no phase separation is obtained as for ABPs [130, 151]. The formation of small clusters, rather than the appearance of MIPS for spherical squirmer, is attributed to changes in the orientational dynamics by interference of the flow fields of the individual squirmers [130, 152]. In contrast, spheroidal squirmers exhibit phase separation and swarming already for rather small activities [130]. Hence, shape and hydrodynamics together govern structure formation of active matter.

\section{Artificial active matter}

Various artificial active agents (motors) have been synthesized during the last decades, ranging from tens of nanometers to micrometers, exploiting diverse propulsion mechanisms [5, 153, 154]. Prominent strategies are based on the slippage of fluid at the surface of the solid particle due to phoretic effects such as diffusiophoresis, involving concentration gradients, thermophoresis, involving thermal gradients, and electrophoresis by inhomogeneities in charge distributions of electrolytes [5, 153-160]. Modeling of artificial active matter aims at a quantitative understanding of the underlying propulsion mechanisms and the design of novel propulsion strategies for practical and technological applications, such as targeted drug delivery.

In general, phoretic effects appear by molecular interactions of a solute in the solution together with the solid particle (motor). Surface reactions generate inhomogeneous concentration fields of reactant and product species in the vicinity of the motor implying concentration gradients over the particle surface. These gradients cause a slippage between the fluid and the particle via diffusiophoresis and induce propulsion of the particle in the fluid. Directed motion appears by controlling the surface reaction, e.g., an asymmetric reaction process on the surface of a Janus particle $[5,153,161]$. The theoretical description of the active process requires to solve the diffusion equation for the concentration of the solute, the hydrodynamic transport problem, and the solid-body equations of motion of the particle. If advection of the solute is neglected, the fluid-chemical transport problem is decoupled an the solute diffusion equation can be solved first. This can then be exploited in the fluid problem to compute the swimming speed and the flow field [158]. The assumption applies at small Péclet numbers, i.e., at small activities and particle sizes, and at large solute diffusion. Contrary, solute advection becomes important at large Péclet numbers and larger particles [162], and significantly impacts the solute velocity [158]. An adequate account of the interactions of the various components involved in phoresis successfully describes the salient features of artificial active particles in solution, e.g., swimming velocity and surface effects, as for Janus particles hovering over or swimming parallel to a surface $[5,163]$.

Response to external fields - Taxis

Biological microswimmers respond to a large variety of external fields by redirecting their motion, in order to locate a target or avoid unfavorable environmental conditions. 
This directed motion is called taxis. Prominent examples are chemo-, photo-, gravi-, magneto-, and rheo-taxis, which describe the response to chemical gradients, light, gravitational, magnetic, and flow fields, respectively. Chemotaxis is used by sperm to find the egg, phototaxis by Chlamydomonas algae to swim toward the light, and rheotaxis by sperm and bacteria to move upstream in flow [164-167]. Modelling and simulation have been employed to understand these phenomena. For example, one mechanism of sperm chemotaxis is the adjustment of the trajectory curvature with a time delay in response to changes in the chemoattractant concentration [168]. It is important to note that chemoand phototaxis in biological system often rely on internal biochemical signalling processes [169, 170].

Redirection of motion in external fields also exists for artificial microswimmers. The mechanisms are usually different, and rely more on direct physical effects. Examples are gravitaxis of chiral (in two dimensions) $L$-shaped microswimmers, which can balance the hydrodynamic and gravitational torques due to shape asymmetry by swimming against gravity [171], collective gravitaxis of bottom-heavy swimmers which form convective swirls in films of finite thickness [172], and phototaxis of thermophoretic colloids which reduce the activity of neighboring colloids by casting a shadow on them [173].

\section{Continuum models for active motion in fluids}

To investigate phenomena occurring at large time and length scales, hydrodynamic theories based on conserved quantities (slow variables) and broken continuous symmetries (order parameters) have been developed, which describe a broad class of systems [174]. Here we discuss a few continuum models of wet active matter, i.e. suspensions of active particles with momentum conservation.

Wet active liquid crystals - Of particular interest are suspensions of active rodlike or elongated objects (e.g. swimming organisms, cytoskeleton, or tissues) embedded in a momentum-conserving solvent and generating active stresses [1-3, 175-177]. The term active gel is also used in the context of the cytoskeleton and tissues, referring to their viscoelastic nature $[175,176]$. Slow variables are the number density $n(\boldsymbol{r}, t)$ of active particles and the total momentum density $\rho \boldsymbol{v}(\boldsymbol{r}, t)$ of the suspension with mass density $\rho$.

Polar active gels consist of particles distinguishing front from rear and are characterized by a polarization field $\boldsymbol{p}(\boldsymbol{r}, t)$ corresponding to the vectorial orientation of active particles. The equations of motion for active gels are derived on the basis of (i) symmetry [178, 179], (ii) irreversible thermodynamics $[175,176,180]$, or (iii) coarse-graining a microscopic theory $[181,182]$. The earliest phenomenological description of wet polar gel $[2,178]$ extends the Toner-Tu model of dry active matter, equation A2, by terms coupling the orientation $\boldsymbol{p}$ to the flow, see equation B3 in Table II. The fluid velocity obeys the incompressible Navier-Stokes equations B1 (NSE), with passive (viscous, elastic and interface) and active contributions to the stress tensor $\sigma$, where the latter is responsible for a spontaneous shear flow without an external stress. The assumption that every swimmer (or molecular motor) exerts an active dipolar force on the solvent (or filament network) yields, to leading order in a gradient expansion, an active stress

$$
\boldsymbol{\sigma}^{a}=-\zeta n(\boldsymbol{r}, t)\left(\boldsymbol{p} \otimes \boldsymbol{p}-\frac{\boldsymbol{p}^{2}}{d} \boldsymbol{I}\right)+O(\nabla),
$$

where $d$ is the dimension, $\boldsymbol{I}$ the unit tensor and $\zeta$ the activity strength, that is $\zeta>0$ for extensile (pushers) and $\zeta<0$ for contractile particles (pullers). Note that $\sigma^{a}$ in equation 5 has nematic symmetry, $\boldsymbol{p} \rightarrow-\boldsymbol{p}$, and active stress with purely polar symmetry arises first in terms containing gradients of $\boldsymbol{p}[181,183]$. The concentration $n$ of active particles evolves via the continuity equation B4.

Active gels of apolar (or head-tail symmetric) particles, called wet active nematics, are described by the nematic alignment tensor field $\boldsymbol{Q}$ introduced in equation A3, where $\boldsymbol{p}$ now denotes the nematic director. The evolution of $\boldsymbol{Q}$ in active nematics at constant concentration $n$ is governed by the nematodynamic equation B5 [90, 184-187] accompanied by the incompressible NSE, equation B1, with an active contribution $\sigma^{a}=-\zeta Q$ to the total stress tensor $\sigma$, cf. equation 5. Note that dynamical equations for active nematics can be obtained from that of polar active gels by interpreting $\boldsymbol{p}$ as nematic director and dropping terms which violate the invariance of the nematic.

Generalized Navier-Stokes equations (GNSE) - Two-fluid models consists of equations for the fluid velocity $\boldsymbol{v}$, the concentration $n$ and the order parameter characterizing the active constituents, like the polarization $\boldsymbol{p}$. For dense suspensions (with constant $n$ ) it is possible to enslave $\boldsymbol{v}$ to $\boldsymbol{p}$ or vice versa (to eliminate one of the vector fields) leading to a simpler one-fluid descriptions of the system [183]. GNSE is a class of minimal, single vector-field models for active fluids like dense microbial suspensions ( $\boldsymbol{v}$ enslaved to $\boldsymbol{p}$, bacterial flow model) [188] or sole passive solvent driven by active components ( $\boldsymbol{p}$ enslaved to $\boldsymbol{v}$, solvent flow model) [189].

In the bacterial flow model, $v$ denotes the velocity of the active subcomponents and GNSE is an extension of the Toner-Tu model: NSE plus a biquadratic Landau velocity potential $F[\boldsymbol{v}]$ (see equations B1 and B6), an active nematic stress contribution via equation B7, and higher order terms in the Fourier expansion of the stress tensor according to equation B8 with $\Gamma_{0}<0, \Gamma_{2}>0$ and $\Gamma_{4}=0$ needed to account for non-local interactions and to reproduce local polar order observed in mesoscale turbulence [188, 190, 191].

GNSE without a velocity potential and the active stress but with additional higher-order stresses via equation B8 with $\Gamma_{0}, \Gamma_{4}>0$ and $\Gamma_{2}<0$, accounting for non-Newtonian effects [189] and originating from active stress with purely polar symmetry [183], describes the solvent dynamics in the presence of active components. Solvent flow model was used to study the rheology of active fluids [192] and active turbulence [183, 193].

Active Model H (AMH)- Active gel models and GNSE consider explicitly or implicitly alignment interactions between active particles. In contrast, AMH describes scalar active matter $(\boldsymbol{p}=\mathbf{Q}=0)$ and is an extension of the active model B (see equations A6, A7 in Table II) to account for the momentumconserving solvent $[194,195]$. Here, the dynamics of the order-parameter field $\phi(\boldsymbol{r}, t)$ obeys the diffusive equations of AMB $[72,195]$ together with an additional advective term $\boldsymbol{v} \cdot \boldsymbol{\nabla} \phi$ coupling $\phi$ to the velocity $\boldsymbol{v}$ of the fluid, whose dynamics is governed by the NSE equations B1. The governing equations of the model are given in equations B9 and B10. 
The violation of the thermodynamic relation between stress $\sigma$ and chemical potential $\mu$ leads to an active contribution to the deviatoric stress (see equation B11); at interfaces the polarization is large $(\boldsymbol{p} \sim \nabla \phi)$ and equation 5 justifies equation B11. The active stress contribution is positive for extensile and negative for contractile swimmers and in the latter case results in an unusual arrested motility-induced phase separation [194].

\section{CELLS AND TISSUES}

Living matter is active matter on all scales: on the protein scale with molecular ATP-consuming machines like motor proteins, ATPase pumps and protein factories, the ribosome; on the cellular scale with cell shape transformation, polarization, migration and division; on the multi-cellular scale with growing biofilms and tissues, tumors, and developing organs; and on the macro-scale with groups, swarms, and herds of animals and humans. The different scales necessitate different model approaches, ranging from all-atom molecular dynamics (MD) simulations for force generating protein machines, over coarse-grained particle-based models and continuum models, to agent-based models with phenomenological interaction rules.

\section{Cytoskeltal filaments and molecular motors}

The ubiquitous microscopic origin of the activity in living matter is the biochemical force generation via energy (ATP) consuming polymerization and depolymerization of cytoskeleton filaments (actin and microtubules) and the collective action of molecular motors. Force generation during polymerization of actin filaments and microtubules is based upon a ratchet mechanism (Box 3): Thermal fluctuations of the target against which the filament polymerizes, e.g., when pushing against the plasma membrane during the formation of filopodia or lemellopodia, allow for the occasional, ATP-dependent, insertion of a new subunit, even when an external force opposes the motion of the target [196, 197]. Asymmetric ATP-dependent polymerization and depolymerization rates at the two ends of cytoskeletal filaments also lead to their effective forward motion called treadmilling $[198,199]$ (Box 3). Both processes are far from equilibrium and at the heart of cell motility.
Force generation of molecular motors [200] has its origin in the massive, ATP-dependent conformational changes that span spatially from the atomic to the molecular level and temporarily up to milli-seconds. All-atom MD simulations are in principle capable of elucidating the underlying molecular processes, while the study of the necessary long time-scale dynamics require efficient sampling techniques and coarse-grained approaches coupled with all-atom MD simulations, termed multiscale MD simulations [201, 202] (note that stability requirements demand time steps of the order of femto-seconds).

To understand the collective behavior of whole ensembles of molecular motors and their interaction with the filament network of the cytoskeleton and the membrane, the number of degrees of freedom has to be reduced drastically. Discrete kinetic and stochastic models for individual motors, as exemplarily sketched in Box 3, yield predictions for the mean velocity and other observables as a function of an imposed load force, the ATP concentration, and other variables $[203,204]$. The effect of the attachment of opposing motors to one cargo are described by a tug-of-war model [205]. Many motors on a single track can lead to molecular motor traffic jams and are described by asymmetric exclusion process (ASEP) models [206] void of any mechano-chemistry.

Particle-based models for cytoskeleton filaments and networks of crosslinked filaments are based on the wormlike chain (WLC) model for semiflexible polymers [207-209]. Computational studies of elastic and collective properties of semiflexible filaments are commonly based on a discretization of the filament into a finite number of segments and subsequent simulation of the Langevin dynamics. The latter is difficult to perform for microtubules since they are a) nearly incompressible in the longitudinal direction, which necessitates the use of constrained Langevin dynamics (fixed segment length) and b) are extremely stiff, which necessitates an extremely small time step in the presence of noise (and which is therefore often neglected). There are several powerful software packages for simulations of biological environments and particle-based modelling of active systems, see list and short description in Supplementary Information, Table S1 [210]. Specific active aspects of microtubule dynamics comprise their length regulation $[199,211]$ and the spindle dynamics during mitosis [212, 213]. 
a| Treadmilling filaments: Subunits are added at the plus-end with rate $v_{a}$. A protein can bind to an empty subunit with rate $\omega_{a}$ and detach with rate $\omega_{d}$. A subunit is removed at the minus-end with rate $v_{d}^{(e)}$ if it does not carry a protein and with rate $v_{d}^{(o)}$ if it does. Mathematically the model is formulated as a master equation for the specified stochastic process. Adapted from ${ }^{199}$.

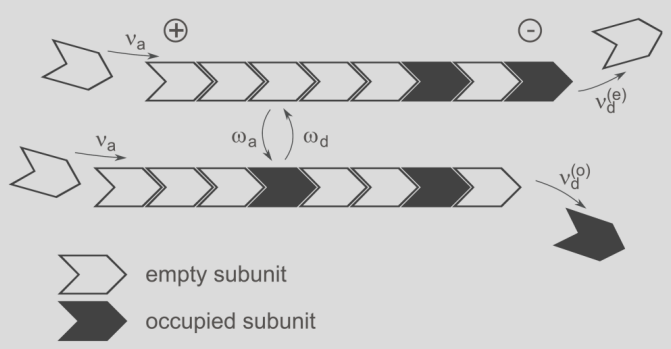

c| Molecular motor models: (left) A schematic representation of the hand-over-hand stepping pattern of a 2-headed motor. (right) A schematic representation of the inchworm stepping pattern of a 2-headed motor. Mathematically the model is formulated as a master equation for the specified stochastic process. Adapted from ${ }^{204}$.
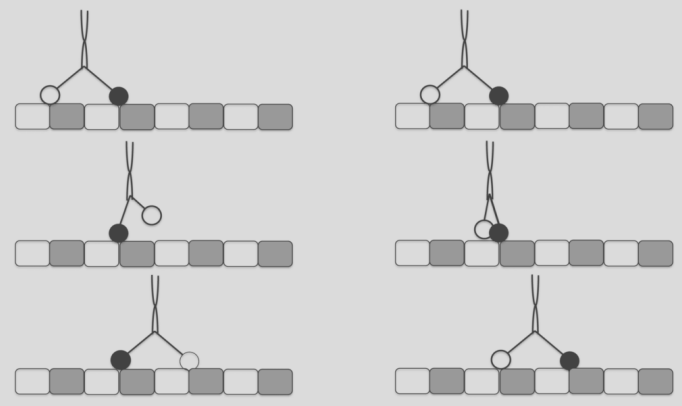

e) Schematic illustration of four cell motility models: (A) G-actin transport to the leading edge creates graded protrusion of the F-actin cytoskeleton. (B) Delivery of new cell membrane via microtubule-assisted vesicle transport controls the rate of protrusion at the leading edge. (C) The RhoGTPases Rac and Rho regulate protrusion (Rac) and contraction (Rho) within the cell. (D) Myosin binds to and contracts the actin cytoskeleton, creating cytoskeletal flows that redistribute the bound myosin. Adapted from ${ }^{223}$.

A G-actin transport model

B Vesicle transport model
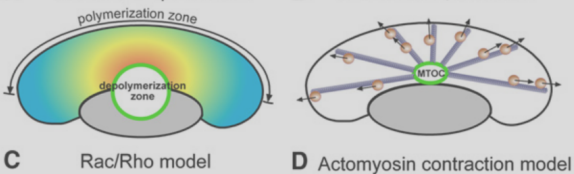

C Rac/Rho model

D Actomyosin contraction model
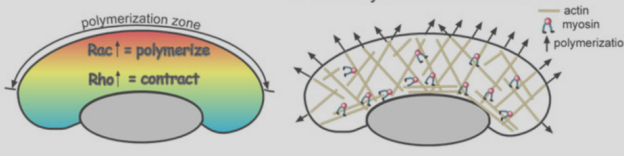

b| Force generation by polymerization of microtubules / actin filaments: The Brownian ratchet principle for polymerization-based (pushing) force generation. Thermal fluctuations of the target allow the occasional insertion (with rate $k_{\text {on }}$ ) of a new subunit (with size $\delta$ ), even when an external force $F_{\text {ext }}$ opposes the motion of the target. Subunits detach with a constant rate $k_{\text {off. }}$ Adapted from ${ }^{197}$.

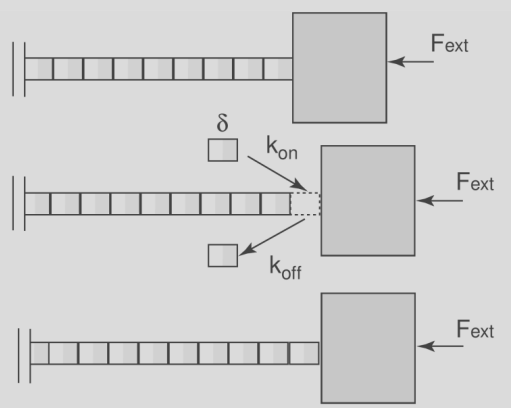

d| Actomyosin as an active gel: Illustration consisting of actin filaments, myosin motors and passive crosslinks (not shown). Filament polymerization and depolymerization processes are indicated by the rates $k_{p}$ and $k_{d}$. Adapted from ${ }^{175}$.

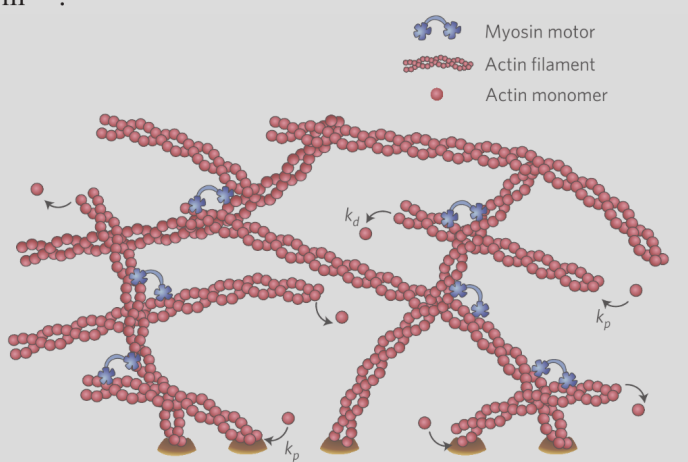

f Cell crawling on substrates: Illustration of the main physical mechanisms responsible for substrate-based cellular motion: actin polymerisation, substrate adhesion and contraction by molecular motors. A minimal phase field model involves a phase field, describing the cell's deformable and movable shape, and a field for the local average orientation of the actin network. The constituting equations contain terms accounting for the pushing force exerted by the actin on the cell boundary, a term for the actin polarization dynamics (essentially localized at the cell boundary), a cell area conservation term, a term describing active stress accounting for the contraction of actomyosin, and a term describing increased motor activity at the rear suppressing the polarization and explicitly breaking the reflection symmetry 224,225 .

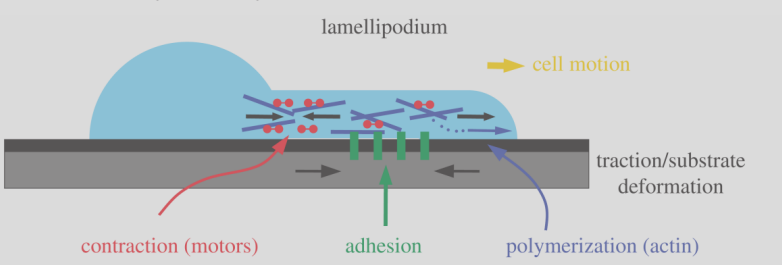

The addition of molecular motors to filament networks (like myosin to actin networks) generates forces that drive the network far from equilibrium and can dramatically alter its stiffness, amplify stress, or lead to network contractility. These effects were studied in an extensible WLC model for semiflexible polymer in which force dipoles were introduced 
into the network at neighboring crosslinks [214, 215], cf. Box 3. On large length and time scales, polymerizing and depolymerizing (treadmilling) actin filaments interconnected by active myosin motors and passive crosslinkers, so called acto-myosin, can be understood as an active gel for which continuum models have been developed, cf. the previous section and Refs. [175, 176, 216, 217]. From this perspective acto-myosin is an active, nematic liquid described by appropriately modified Navier-Stokes equations: the central quantity is the stress tensor which depends on the velocity gradient, the orientational field of the filaments, and an active stress generated by the motors and actin polymerization. Since cytoskeletal filaments can act as tracks to motors and in turn motors can move filaments, the active stress is coupled to the orientational field. Within this active hydrodynamics framework cell locomotion and cellular shape changes and many experimental situations have been successfully discussed $[175,176]$.

\section{Cell motility models}

The central molecular machinery that enables eukaryotic cells to spontaneously move is the actin cytoskeleton responsible for cellular shape changes, like the formation of thin membrane-bound protrusions. Thus, in addition to a mathematical representation of the acto-myosin machinery, cell-motility models must involve a representation of the cell membrane and a description of adhesion and force transmission to a substrate or a three-dimensional environment [198, 218-221]. In the paradigmatic model of cell crawling on substrates, migration is divided into discrete steps: (a) protrusion based on actin growth and polymerization force; (b) formation of new adhesions at the front; (c) release and recycling of adhesions at the rear; and finally, (d) actinmyosin-powered contraction of the cytoplasm, resulting in forward translocation of the cell body [220], cf. Box 3. In a model that relies mainly on actin treadmilling and diffusing actin nucleators, cell crawling is driven by actin polymerization waves without motors [222].

The major technical challenge for a continuum modeling of cell migration is the presence of a moving boundary and the nonlinear and nonlocal coupling of cytoskeletal dynamics to a moving and deformable membrane. Various continuum models for cell motility on substrates have been employed [219, 222-226]. Commonly, three different modeling approaches have been used: sharp-interface models, in which the interface is represented by a curve that moves with some velocity, level set methods, and diffuse-interface models. In the latter two descriptions, a phase field distinguishes the two phases (the interior and the exterior of the cell), where either the zero contour of the phase field determines the position of the membrane, or there is a gradual variation of the different physical quantities across the interface [227]. Phase-field models have also been used for multi-cellular systems [228, 229], as for collective cell migration [230] (for a review about physical models for collective cell motility see [231]) and tissues [232, 233], see also Box 4.

Alternatively, microscopic models with explicit membrane and self-propelled (pulling or pushing) filaments can be employed [234, 235]. This approach incorporates fluctuations of the internal structure, persistent and random-walk-like motion, and shape changes in response to external conditions.

\section{Tissues}

Tissues are aggregates of adherent cells, sometimes organized in layers (e.g., epithelia). In addition to constitute a viscoelasto-plastic material [236], tissues generate actively internal tension via cell proliferation and death, as for instance during growth, and generate active stress by cellular force generation, as during muscle contraction. The technical challenge for a continuum formulation of volumetric growth in soft elastic tissues is the persistent change of the equilibrium configurations against which small deformations must be defined [237].

Particle-based models for tissue growth represent cells as spheres that continuously deform into dumbbells until division occurs [238, 239], cf. Box 4. The particles representing cells can adhere to each other, maintain volume exclusion, exert an active growth pressure on their surrounding, expand in size until reaching a size checkpoint, divide when reaching this checkpoint size, undergo apoptosis, exert random forces on neighboring cells, regulate to their homeostatic state via cell division and apoptosis in a confined volume, and comply with force balance and momentum conservation. Either Langevin dynamics [238] or dissipative particle dynamics [238, 239] have been employed as constitutive dynamics. Simulations show that stress-induced growth inhibition is responsible for the transition from exponential to sub-exponential growth experimentally observed in tumor spheroids, and lack of nutrients determines the size of the necrotic core but not the size of the tumor [238]. Moreover, cell division and apoptosis also lead to a fluidization of the tissue [239-241] as further analyzed in a continuum theory described below. 


\section{Box 4| Models for tissue growth}

a| Cellular Potts model (CPM): (a) Individual cells are (a) represented by a connected set of lattice unit cells, red hexagons for cell $\alpha$ and blue for cell $\beta$. Membrane sites of $\alpha$ are indicated with a grey shade, the immediate neighborhood by a dashed line. (b) Sketch of examples for elementary protrusion and retraction events. For grid sites within range $\mathrm{R}$ of an accepted elementary event, here indicated by + or -, the regulatory factors are incremented or decremented. The cell configurations evolve stochastically by updating lattice unit cells with probabilities derived form a "Hamiltonian", which takes into account cell contractility, cell-cell adhesion and cytoskeletal remodeling. Adapted from ${ }^{249}$.

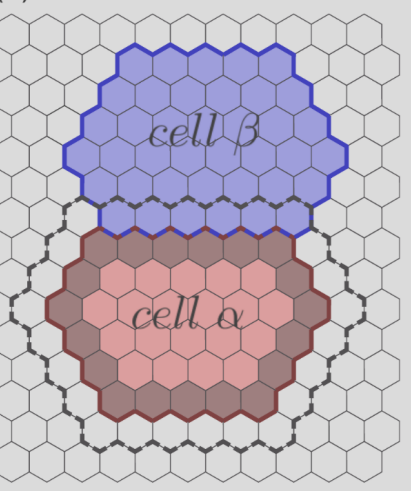

(b) protrusion

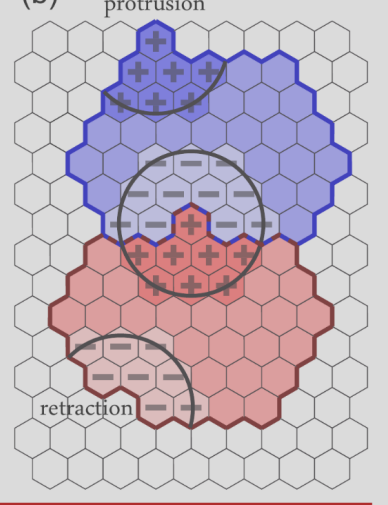

b| Vertex model for confuent monolayers: A polygonal tiling of space represents the conf uent monolayer, where each polygon corresponds to a cell. Polygons are updated by moving their vertices using deterministic equations of motions $\eta \mathrm{d} \mathbf{x}_{i}(t) / \mathrm{d} t=\mathbf{F}_{i}$, where $\mathbf{x}_{i}$ is the position of vertex $i$, as exemplifed in the left fgure, and a set of graph changing rules as indicated in the right $f$ gure, e.g. cell neighbor exchange (upper right sketch) or division (lower right sketch). The dynamics is thus a combination of relaxation to mechanical equilibrium and changes in tissue connectivity. Adapted from ${ }^{251}$.

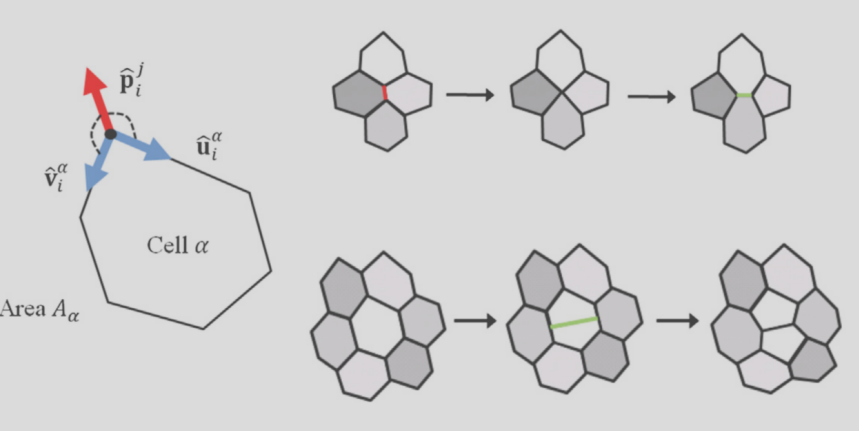

c| Particle based tissue growth model: The tissue is described by an ensemble of interacting cells, where a cell is represented by a dumbbell (see sketch), which can increase the distance between the two spheres, representing growth, and can split into two spheres, representing division (dissipative particle dynamics simulations). In addition, propulsion as well as repulsive/attractive forces can be taken into account by conservative interactions. Adapted from ${ }^{66,238}$.

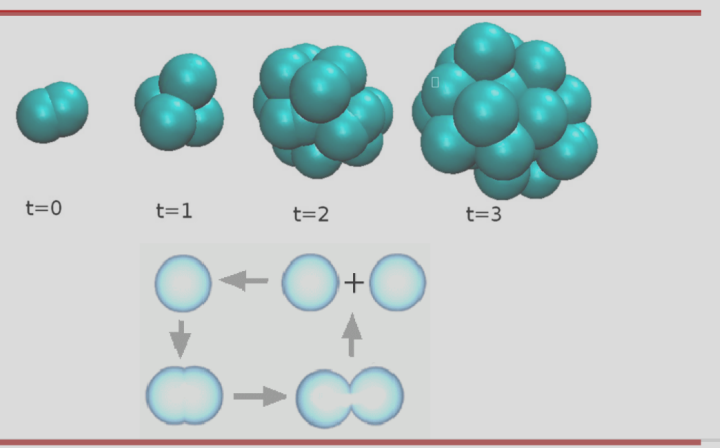

d| Multicellular phase field models: The shape of a cell $i$ is defined by a phase field $\varphi_{i}(x)$ varying between 0 and 1. Its dynamics is defined by a set of equations analogous to the phase field description of single cell migration. The repulsion of two cells $i$ and $j$ involves the product $\varphi_{i}(x) \cdot \varphi_{j}(x)$, which is non-zero only where the two phase fields overlap, see sketch. Adapted from ${ }^{231}$.

Alternatively, lattice-based models for tissue growth and morphogenesis have been extensively employed. The cel- lular Potts model (CPM) [242-244] defines the cell shape with the help of discrete variables assigned to each site of 
a regular lattice, cf. Box 4. An energy function regulates cell volume, cell surface area, cell adhesion, etc. The CPM has been applied to development [245] and vasculogenesis [246], but also to cell migration [247] and cell shape dynamics on micropatterned surfaces [248, 249]. Another lattice model is the confluent tissue vertex or Voronoi model [250-253], where confluent monolayers are represented as a polygonal tiling of space and each polygon corresponds to a cell, cf. Box 4. These models have been used to study the jamming transition in tissues [254] with a CPM [255] and a Voronoi [256] model.

Continuum models for tissue mechanics have a long tradition in the field of Biomechanics and Biomedical Engineering [257]. A major challenge in continuum models for tissue growth is the coupling between growth rate and local stress, which is modeled by a dependence of the growth-rate tensor on the stress tensor [237, 258, 259]. A simple lowdimensional example is the mathematical model of nonuniform growth in a monolayer [260], which incorporates a mechanical feedback mechanism via an explicit dependence of the local tissue growth rate on the degree of local compression (or stretching) of the tissue. In Ref. [66], threedimensional tissues were considered as elastic media and it was shown that the coupling of cell division and cell death to the local stresses effectively leads to viscoelastic behavior with a relaxation time set by the rate of cell division.

It should also be mentioned that various continuum models for tumor growth are based on the theory of mixtures [261] as reviewed in Ref. [262]. Since tissue growth in a living organism requires nutrition supply and oxygen, a blood vessel network should be included: Hybrid models describe the tumor mass with a continuum model and the dynamically changing vascularization with a discrete pipe network [263, 264]. Simulations show that the incorporation of a blood vessel network leads to a characteristic compartmentalization of the tumor into several regions differing in vessel density and diameter, and in necrosis.

\section{ANIMAL GROUPS}

Collective migration is of paramount importance for a wide variety of animals, such as swarms of insects, fish schools, bird flocks, herds of game, and human crowds. The models of dry active matter (cf. Table I), in particular the Vicsek model and its extensions [52], capture prototypical aspects of the collective behavior of such animal groups. Despite of sharing some universal features, the observed motion patterns differ substantially among animal groups due to differences in the nature of interactions between individuals, which requires adaptation of basic models. Important characteristics include social behavior mediated by chemical, acoustic, or optical signals. Chemical signaling comprises non-reciprocal attractive-repulsive interactions, which imply specific actions such as pursuit-escape behavior [265-267]. Particular attention has been paid to modeling vision-based interactions, where the emerging motion patterns depend on the field of view [268-270].

Various strategies are applied to unravel the mechanisms underlying the collective behavior of animal groups. In a phenomenological top-down approach, (physical) interactions are deduced from observations [270, 271]. Alternatively, in a bottom-up approach, the effect of complex information processing strategies of individual agents, e.g., delayed signal processing, is studied [272-274]. Collective behavior can even emerge from a purely probabilistic approach by considering intrinsic motivation and maximization of future options via processing of sensed information, without any a priori specification of social forces or individual interaction rules [273, 275].

Living active agents travelling together have evolutionary developed efficient decision-making mechanisms to search, forage, escape, etc. For instance quorum sensing, i.e., the ability of individuals to coordinate their activities according to the local population density, facilitates information transfer in these systems [269, 276, 277]. While such cooperative interactions are powerful tools to reach collective decisions [278], in some cases only a few individuals possess the required knowledge about the migration route, location of food sources, etc. [279]. Accordingly, numerical models have been studied to understand the mechanisms of effective leadership in biological systems, and the impact of the density of informed individuals and their communications on the information transfer in animal groups [280-282]. A realistic description of collective human motion is crucial to prevent crowd disasters [283-285].

\section{OUTLOOK AND FUTURE DIRECTIONS}

A powerful pool of computational models for active matter, particle-based or field-theoretical, have been developed in the recent years (see a list of computational methods for active matter in Table III). This has lead to an enormous knowledge gain for the non-equilibrium behavior of active systems. Yet, many challenges remain.

The statistical-physics description of active systems needs to be extended in several directions, like the incorporation of an activity-dependent noise to account for the stochastic nature of active forces, or the generalization of field-theoretical models to systems far from equilibrium. Current field theories are based on a linear expansion of fluxes in terms of forces and can therefore in principle describe only systems close to equilibrium; however, biological systems are typically far from equilibrium and there is no comprehensive continuum theory for systems far from equilibrium yet.

The investigation of the collective behavior of selfpropelled particles is limited so far to relatively simple systems. In particular, the interplay of different interactions, such as shape and hydrodynamics, of complex environments, such as viscoelastic fluids and intricate confining geometries, external fields, such as gravity and turbulent flows, mixtures of active and passive particles, as well as information exchange by vision-like interactions, holds many unexpected surprises to be discovered.

A plethora of models for cell motility has been employed in studies of migration on two-dimensional substrates. However, many cells in living organisms move in three-dimensional (3D) environments, like the extra-cellular matrix (ECM), and there is a lack of continuum models for motility in 3D. First studies have focused on rigidity sensing and subsequent polarization of the cell toward stiffer ECM [286]. Furthermore, for migration in 3D environments, cells can utilize other modes of motility in addition to adhesionbased lamellipodial propulsion, in particular blebbing motility, which is based on the formation of blebs at the leading 
Molecular Dynamics (MD)

Standard approach to solve Newton's equations of motion. For active matter, energy input and a thermostat is required to drive the system out of equilibrium and to allow for a stationary state for long times.

Brownian Dynamics (BD/LD)

Particle dynamics is described by the overdamped Newton's equations of motion with active force and with/without thermal noise.

Kinetic Monte Carlo (MC)

A series of Monte Carlo steps is performed for the configurational variables with an acceptance probability, e.g., obtained from importance sampling with the energy difference between original and attempted new state. Usually applied to lattice tissue-growth models like the cellular Potts model.

Lattice Boltzmann (LB) Mesoscale hydrodynamics approach, which is based on propagating velocity-distribution functions on lattices. Standard version without thermal fluctuations, therefore well suited for larger microswimmers.

Particle-based mesoscale hydrodynamics approach, which is a generalisation of

Dissipative Particle Dynamics (DPD) Molecular Dynamics by including pairwise friction and noise forces. Multi-phase flows easily incorporated by direct conservative interactions between different particle species. Active particles coupled to fluid by non-conservative forces.

Particle-based mesoscale hydrodynamics approach, which employs an ensemble of point particles for the fluid with multi-particle collisions in a regular lattice of collision boxes. Naturally includes thermal fluctuations. Avoids depletion interactions between active solutes, therefore, well suited for active systems with small characteristic length scales.

Hydrodynamic interactions integrated out by the use of Greens' functions of Stokes

Boundary Integral Method (BIM) equation in favor of forces between boundary elements of walls and embedded active particles. Active systems modeled by non-conservative boundary forces.

Solution of the Navier-Stokes equations on a lattice by finite element/volume methods, where all spatial scales are resolved in the computational mesh. Coupling to active particles via boundary conditions.

Direct Numerical Simulation (DNS

The effect of active fluids on embedded passive particles is different from thermal fluctuations due to finite persistence times, which can be captured by memory kernels or colored noise.

Colored Noise

Lattice-based model for the dynamics of cell populations and tissues ( $2 \mathrm{~d}$ and $3 \mathrm{~d}$ ). Useful when the shape and size of individual cells and the contact area between neighboring cells determine the intercellular interactions. Individual cells are represented by multiple connected lattice sites, which are updated stochastically via Kinetic Monte Carlo method.

Cellular Potts Model

Discrete model in continuum space for the dynamics of confluent cell monolayers (2d). Useful when the shape and size of individual cells and the contact area between neighboring cells determine the intercellular interactions. The confluent monolayer is represented as a polygonal tiling of space where each polygon corresponds to a cell. Polygons are updated by moving their vertices using deterministic equations of motions and a set of graph changing rules.

Vertex Mode

Continuum model to describe the dynamics of individual cells and of cell populations. Useful to describe actin driven cell migration and shape transformations. Individual cells are represented by a phase field, which interacts with other fields like the local actin polarization. Tissues are described by multiple phase fields, each corresponding to a cell. The interaction between two neighboring cells is determined by the overlap between their phase fields and the spatio-temporal evolution is defined by partial differential equations.

TABLE III | Computational methods for simulation of active matter.

edge (blebs emerge when the plasma membrane of a cell delaminates locally from its actin cortex, leading to pressuredriven protrusions, followed by membrane re-attachment) [287]. Again continuum modeling has received little attention so far. A first modeling study for blebbing-induced migration in 2D still crucially depends on protrusions adhering to a substrate [288].

Finally, signalling is a very important aspect of cell motility, tissue growth, the development of bacterial colonies, and the collective motion of animal herds, which has been neglected in most computational models for active matter so far. Challenges include the modeling of the interplay of biochemistry and mechanics on a single cell level [289-291]), and the reaction to external factors like food, smell, or light (sun) in flocks, schools, and herds. Quantitative models for tissue growth, morphogenesis, biofilm formation, wound healing, or the cancer growth and metastastisis need to include biochemical signaling coupled to mechanical stress. Similarly, realistic models for human groups, car or pedestrian traffic need to take into account heterogeneous behavior and varying reactions of individuals to external cues, which also asks for the investigation of a putative leader role.

An overarching challenge for the whole field is the distinction between the generic and specific properties and behavior of a particular active-matter system. What are the universal properties shared by a large class of systems, and when do specific mechanisms of propulsion or interactions come into play and dominate the behavior?

\section{Acknowledgements}

M.R.S., A.W., and H.R. acknowledge support by DFG within SFB 1027 (A3, A7). R. G. W. and G. G. acknowledge funding by DFG within the priority program SPP 1726 "Microswimmers - from Single Particle Motion to Collective Behavior".

\section{Author contributions}

All authors contributed to all aspects of manuscript preparation, revision and editing. Request for materials: h.rieger@mx.uni-saarland.de or shaebani@lusi.uni-sb.de. The authors declare no competing interests.

\section{References}


1. Marchetti, M. C., Joanny, J. F., Ramaswamy, S., Liverpool, T. B., Prost, J., Rao, M. \& Simha, R. A. Hydrodynamics of soft active matter. Rev. Mod. Phys. 85, 1143-1189 (2013).

2. Ramaswamy, S. The mechanics and statistics of active matter. Annu. Rev. Condens. Matter Phys. 1, 323-345 (2010).

3. Toner, J., Tu, Y. \& Ramaswamy, S. Hydrodynamics and phases of flocks. Ann. Phys. 318, 170-244 (2005).

4. Elgeti, J., Winkler, R. G. \& Gompper, G. Physics of microswimmers-single particle motion and collective behavior: a review. Rep. Prog. Phys. 78, 056601 (2015).

5. Bechinger, C., Di Leonardo, R., Löwen, H., Reichhardt, C., Volpe, G. \& Volpe, G. Active particles in complex and crowded environments. Rev. Mod. Phys. 88, 045006 (2016).

6. Romanczuk, P., Bär, M., Ebeling, W., Lindner, B. \& SchimanskyGeier, L. Active Brownian particles. Eur. Phys. J. Spec. Top. 202, 1-162 (2012).

7. Nguyen, N. H. P., Klotsa, D., Engel, M. \& Glotzer, S. C. Emergent collective phenomena in a mixture of hard shapes through active rotation. Phys. Rev. Lett. 112, 075701 (2014).

8. Löwen, H. Chirality in microswimmer motion: From circle swimmers to active turbulence. Eur. Phys. J. Spec. Top. 225, 2319-2331 (2016).

9. Peruani, F. Active Brownian rods. Eur. Phys. J. Spec. Top. 225, 2301-2317 (2016).

10. ten Hagen, B., Wittkowski, R., Takagi, D., Kümmel, F., Bechinger, C. \& Löwen, H. Can the self-propulsion of anisotropic microswimmers be described by using forces and torques? J. Phys. Condens. Matter 27, 194110 (2015).

11. Kaiser, A., Babel, S., ten Hagen, B., von Ferber, C. \& Löwen, H. How does a flexible chain of active particles swell? $J$. Chem. Phys. 142, 124905 (2015).

12. Eisenstecken, T., Gompper, G. \& Winkler, R. G. Conformational properties of active semiflexible polymers. Polymers $\mathbf{8}$, 304 (2016).

13. Eisenstecken, T., Gompper, G. \& Winkler, R. G. Internal dynamics of semiflexible polymers with active noise. J. Chem. Phys. 146, 154903 (2017).

14. Kourbane-Houssene, M., Erignoux, C., Bodineau, T. \& Tailleur, J. Exact hydrodynamic description of active lattice gases. Phys. Rev. Lett. 120, 268003 (2018).

15. Klamser, J. U., Kapfer, S. C. \& Krauth, W. Thermodynamic phases in two-dimensional active matter. Nat. Commun. 9, 5045 (2018).

16. Sadjadi, Z., Shaebani, M. R., Rieger, H. \& Santen, L. Persistent-random-walk approach to anomalous transport of self-propelled particles. Phys. Rev. E 91, 062715 (2015).

17. Shaebani, M. R., Sadjadi, Z., Sokolov, I. M., Rieger, H. \& Santen, L. Anomalous diffusion of self-propelled particles in directed random environments. Phys. Rev. E 90, 030701 (2014).

18. Levis, D. \& Berthier, L. Clustering and heterogeneous dynamics in a kinetic Monte Carlo model of self-propelled hard disks. Phys. Rev. E 89, 062301 (2014).

19. Najafi, J., Shaebani, M. R., John, T., Altegoer, F., Bange, G. \& Wagner, C. Flagellar number governs bacterial spreading and transport efficiency. Science Adv. 4 (2018).

20. Hafner, A. E., Santen, L., Rieger, H. \& Shaebani, M. R. Runand-pause dynamics of cytoskeletal motor proteins. Sci. Rep. 6, 37162 (2016)

21. Redner, G. S., Hagan, M. F. \& Baskaran, A. Structure and dynamics of a phase-separating active colloidal fluid. Phys. Rev. Lett. 110, 055701 (2013).

22. Cates, M. E. \& Tailleur, J. Motility-induced phase separation. Annu. Rev. Condens. Matter Phys. 6, 219-244 (2015).

23. Wysocki, A., Winkler, R. G. \& Gompper, G. Cooperative motion of active Brownian spheres in three-dimensional dense suspensions. Europhys. Lett. 105, 48004 (2014).

24. Stenhammar, J., Marenduzzo, D., Allen, R. J. \& Cates, M. E. Phase behaviour of active Brownian particles: the role of dimensionality. Soft Matter 10, 1489-1499 (2014).

25. Wysocki, A., Winkler, R. G. \& Gompper, G. Propagating interfaces in mixtures of active and passive Brownian particles. New J. Phys. 18, 123030 (2016).

26. Stenhammar, J., Wittkowski, R., Marenduzzo, D. \& Cates, M. E. Activity-induced phase separation and self-assembly in mixtures of active and passive particles. Phys. Rev. Lett. 114, 018301 (2015).

27. Digregorio, P., Levis, D., Suma, A., Cugliandolo, L. F., Gonnella, G. \& Pagonabarraga, I. Full phase diagram of active Brownian disks: From melting to motility-induced phase separation. Phys. Rev. Lett. 121, 098003 (2018).

28. Fily, Y., Henkes, S. \& Marchetti, M. C. Freezing and phase separation of self-propelled disks. Soft Matter 10, 2132-2140 (2014).

29. Elgeti, J. \& Gompper, G. Wall accumulation of self-propelled spheres. EPL 101, 48003 (2013).

30. Fily, Y., Baskaran, A. \& Hagan, M. Dynamics of self-propelled particles under strong confinement. Soft Matter 10, 56095617 (2014).

31. Das, S., Gompper, G. \& Winkler, R. G. Local stress and pressure in an inhomogeneous system of spherical active Brownian particles. Sci. Rep. 9, 6608 (2019).

32. Wysocki, A. \& Rieger, H. Capillary action in scalar active matter. arXiv e-prints arXiv:1908.03368 (2019).

33. Takatori, S. C., Yan, W. \& Brady, J. F. Swim pressure: Stress generation in active matter. Phys. Rev. Lett. 113, 028103 (2014).

34. Solon, A. P., Fily, Y., Baskaran, A., Cates, M. E., Kafri, Y., Kardar, M. \& Tailleur, J. Pressure is not a state function for generic active fluids. Nat. Phys. 11, 673 (2015).

35. Winkler, R. G., Wysocki, A. \& Gompper, G. Virial pressure in systems of active Brownian particles. Soft Matter 11, 66806691 (2015).

36. Fily, Y., Kafri, Y., Solon, A. P., Tailleur, J. \& Turner, A. Mechanical pressure and momentum conservation in dry active matter. J. Phys. A: Math. Theor. 51, 044003 (2018).

37. Wysocki, A., Elgeti, J. \& Gompper, G. Giant adsorption of microswimmers: Duality of shape asymmetry and wall curvature. Phys. Rev. E 91, 050302(R) (2015).

38. Tjhung, E., Nardini, C. \& Cates, M. E. Cluster phases and bubbly phase separation in active fluids: Reversal of the ostwald process. Phys. Rev. X 8, 031080 (2018).

39. Bratanov, V., Jenko, F. \& Frey, E. New class of turbulence in active fluids. Proc. Natl. Acad. Sci. USA 112, 15048-15053 (2015).

40. Weber, C. A., Hanke, T., Deseigne, J., Léonard, S., Dauchot, O., Frey, E. \& Chaté, H. Long-range ordering of vibrated polar disks. Phys. Rev. Lett. 110, 208001 (2013).

41. Hu, J., Yang, M., Gompper, G. \& Winkler, R. G. Modelling the mechanics and hydrodynamics of swimming e. coli. Soft Matter 11, 7867-7876 (2015).

42. Vicsek, T. \& Zafeiris, A. Collective motion. Phys. Rep. 517, 71-140 (2012).

43. Vicsek, T., Czirók, A., Ben-Jacob, E., Cohen, I. \& Shochet, O. Novel type of phase transition in a system of self-driven particles. Phys. Rev. Lett. 75, 1226-1229 (1995).

44. Aldana, M., Dossetti, V., Huepe, C., Kenkre, V. M. \& Larralde, $\mathrm{H}$. Phase transitions in systems of self-propelled agents and related network models. Phys. Rev. Lett. 98, 095702 (2007).

45. Aldana, M., Larralde, H. \& Vazquez, B. On the emergence of collective order in swarming systems: A recent debate. Int. J. Mod. Phys. B 23, 3661-3685 (2009).

46. Peruani, F. \& Aranson, I. S. Cold active motion: How timeindependent disorder affects the motion of self-propelled agents. Phys. Rev. Lett. 120, 238101 (2018).

47. Grossman, D., Aranson, I. S. \& Jacob, E. B. Emergence of agent swarm migration and vortex formation through inelastic collisions. New J. Phys. 10, 023036 (2008).

48. Nagy, M., Daruka, I. \& Vicsek, T. New aspects of the continuous phase transition in the scalar noise model (snm) of collective motion. Physica A 373, 445-454 (2007).

49. Peruani, F., Klauss, T., Deutsch, A. \& Voss-Boehme, A. Traffic 
jams, gliders, and bands in the quest for collective motion of self-propelled particles. Phys. Rev. Lett. 106, 128101 (2011).

50. Ginelli, F. \& Chaté, H. Relevance of metric-free interactions in flocking phenomena. Phys. Rev. Lett. 105, 168103 (2010).

51. Gregoire, G., Chaté, H. \& Tu, Y. Moving and staying together without a leader. Physica D 181, 157-170 (2003).

52. Chaté, H., Ginelli, F., Grégoire, G., Peruani, F. \& Raynaud, F. Modeling collective motion: variations on the vicsek model. Eur. Phys. J. B 64, 451-456 (2008).

53. Szabó, P., Nagy, M. \& Vicsek, T. Transitions in a self-propelledparticles model with coupling of accelerations. Phys. Rev. E 79, 021908 (2009).

54. Chaté, H., Ginelli, F. \& Montagne, R. Simple model for active nematics: Quasi-long-range order and giant fluctuations. Phys. Rev. Lett. 96, 180602 (2006).

55. Mahault, B., Jiang, X.-c., Bertin, E., Ma, Y.-q., Patelli, A., Shi, X.-q. \& Chaté, H. Self-propelled particles with velocity reversals and ferromagnetic alignment: Active matter class with second-order transition to quasi-long-range polar order. Phys. Rev. Lett. 120, 258002 (2018).

56. Ginelli, F., Peruani, F., Bär, M. \& Chaté, H. Large-scale collective properties of self-propelled rods. Phys. Rev. Lett. 104, 184502 (2010).

57. Strömbom, D. Collective motion from local attraction. J. Theor. Biol. 283, 145-151 (2011).

58. Solon, A. P., Chaté, H. \& Tailleur, J. From phase to microphase separation in flocking models: The essential role of nonequilibrium fluctuations. Phys. Rev. Lett. 114, 068101 (2015).

59. Solon, A. P. \& Tailleur, J. Revisiting the flocking transition using active spins. Phys. Rev. Lett. 111, 078101 (2013).

60. Szabó, B., Szöllösi, G. J., Gönci, B., Jurányi, Z., Selmeczi, D. \& Vicsek, T. Phase transition in the collective migration of tissue cells: Experiment and model. Phys. Rev. E 74, 061908 (2006).

61. Peruani, F., Deutsch, A. \& Bär, M. Nonequilibrium clustering of self-propelled rods. Phys. Rev. E 74, 030904 (2006).

62. Toner, J. \& Tu, Y. Long-range order in a two-dimensional dynamical XY model: How birds fly together. Phys. Rev. Lett. 75, 4326-4329 (1995).

63. Toner, J. \& Tu, Y. Flocks, herds, and schools: A quantitative theory of flocking. Phys. Rev. E 58, 4828-4858 (1998).

64. Bertin, E., Droz, M. \& Grégoire, G. Boltzmann and hydrodynamic description for self-propelled particles. Phys. Rev. E 74, 022101 (2006)

65. Ihle, T. Kinetic theory of flocking: Derivation of hydrodynamic equations. Phys. Rev. E 83, 030901 (2011).

66. Ranft, J., Basan, M., Elgeti, J., Joanny, J.-F., Prost, J. \& Jülicher, F. Fluidization of tissues by cell division and apoptosis. Proc. Natl. Acad. Sci. USA 107, 20863-20868 (2010).

67. Narayan, V., Ramaswamy, S. \& Menon, N. Long-lived giant number fluctuations in a swarming granular nematic. Science 317, 105-108 (2007).

68. Ramaswamy, S., Simha, R. A. \& Toner, J. Active nematics on a substrate: Giant number fluctuations and long-time tails. EPL 62, 196-202 (2003).

69. Hemingway, E. J., Maitra, A., Banerjee, S., Marchetti, M. C., Ramaswamy, S., Fielding, S. M. \& Cates, M. E. Active viscoelastic matter: From bacterial drag reduction to turbulent solids. Phys. Rev. Lett. 114, 098302 (2015).

70. Schwarz, U. S. \& Safran, S. A. Physics of adherent cells. Rev. Mod. Phys. 85, 1327-1381 (2013).

71. Hohenberg, P. C. \& Halperin, B. I. Theory of dynamic critical phenomena. Rev. Mod. Phys. 49, 435-479 (1977).

72. Wittkowski, R., Tiribocchi, A., Stenhammar, J., Allen, R. J., Marenduzzo, D. \& Cates, M. E. Scalar $\phi^{4}$ field theory for active-particle phase separation. Nat. Commun. 5, 4351 (2014).

73. Stenhammar, J., Tiribocchi, A., Allen, R. J., Marenduzzo, D. \& Cates, M. E. Continuum theory of phase separation kinetics for active Brownian particles. Phys. Rev. Lett. 111, 145702 (2013).
74. Cates, M. E. \& Tailleur, J. When are active Brownian particles and run-and-tumble particles equivalent? consequences for motility-induced phase separation. EPL 101, 20010 (2013).

75. Tailleur, J. \& Cates, M. E. Statistical mechanics of interacting run-and-tumble bacteria. Phys. Rev. Lett. 100, 218103 (2008).

76. Purcell, E. M. Life at low Reynolds number. Am. J. Phys. 45, 3-11 (1977).

77. Spagnolie, S. E. \& Lauga, E. Hydrodynamics of self-propulsion near a boundary: predictions and accuracy of far-field approximations. J. Fluid Mech. 700, 105-147 (2012).

78. Winkler, R. G. \& Gompper, G. Hydrodynamics in motile active matter. In Andreoni, W. \& Yip, S. (eds.) Handbook of Materials Modeling: Methods: Theory and Modeling, 120 (Springer International Publishing, Cham, 2018). URL https://link.springer.com/referencework/.

79. Berke, A. P., Turner, L., Berg, H. C. \& Lauga, E. Hydrodynamic attraction of swimming microorganisms by surfaces. Phys. Rev. Lett. 101, 038102 (2008).

80. Lauga, E. \& Powers, T. R. The hydrodynamics of swimming microorganisms. Rep. Prog. Phys. 72, 096601 (2009).

81. Elgeti, J. \& Gompper, G. Microswimmers near surfaces. Eur. Phys. J. Spec. Top. 225, 2333-2352 (2016).

82. Li, G. \& Tang, J. X. Accumulation of microswimmers near a surface mediated by collision and rotational Brownian motion. Phys. Rev. Lett. 103, 078101 (2009).

83. Elgeti, J. \& Gompper, G. Self-propelled rods near surfaces. EPL 85, 38002 (2009).

84. Elgeti, J. \& Gompper, G. Run-and-tumble dynamics of selfpropelled particles in confinement. EPL 109, 58003 (2015).

85. Schaar, K., Zöttl, A. \& Stark, H. Detention times of microswimmers close to surfaces: Influence of hydrodynamic interactions and noise. Phys. Rev. Lett. 115, 038101 (2015).

86. Saintillan, D. \& Shelley, M. J. Orientational order and instabilities in suspensions of self-locomoting rods. Phys. Rev. Lett. 99, 058102 (2007).

87. Saintillan, D. \& Shelley, M. J. Instabilities and pattern formation in active particle suspensions: Kinetic theory and continuum simulations. Phys. Rev. Lett. 100, 178103 (2008).

88. Sanchez, T., Chen, D. T. N., DeCamp, S. J., Heymann, M. \& Dogic, Z. Spontaneous motion in hierarchically assembled active matter. Nature 491, 431-434 (2012).

89. Thampi, S. P., Golestanian, R. \& Yeomans, J. M. Velocity correlations in an active nematic. Phys. Rev. Lett. 111, 118101 (2013).

90. Giomi, L., Bowick, M. J., Ma, X. \& Marchetti, M. C. Defect annihilation and proliferation in active nematics. Phys. Rev. Lett. 110, 228101 (2013).

91. Keber, F. C., Loiseau, E., Sanchez, T., DeCamp, S. J., Giomi, L., Bowick, M. J., Marchetti, M. C., Dogic, Z. \& Bausch, A. R. Topology and dynamics of active nematic vesicles. Science 345, 1135-1139 (2014).

92. Mathijssen, A. J. T. M., Culver, J., Bhamla, M. S. \& Prakash, M. Collective intercellular communication through ultra-fast hydrodynamic trigger waves. Nature 571, 560-564 (2019).

93. Qiu, T., Lee, T.-C., Mark, A. G., Morozov, K. I., Münster, R., Mierka, O., Turek, S., Leshansky, A. M. \& Fischer, P. Swimming by reciprocal motion at low Reynolds number. Nat. Commun. 5, 5119 (2014)

94. Qin, B., Gopinath, A., Yang, J., Gollub, J. P. \& Arratia, P. E. Flagellar kinematics and swimming of algal cells in viscoelastic fluids. Sci. Rep. 5, 9190 (2015).

95. Patteson, A. E., Gopinath, A., Goulian, M. \& Arratia, P. E. Running and tumbling with e. coli in polymeric solutions. Sci. Rep. 5, 15761 (2015).

96. Li, G. \& Ardekani, A. M. Collective motion of microorganisms in a viscoelastic fluid. Phys. Rev. Lett. 117, 118001 (2016).

97. Lauga, E. Propulsion in a viscoelastic fluid. Phys. Fluids 19, 083104 (2007).

98. Fu, H. C., Wolgemuth, C. W. \& Powers, T. R. Swimming speeds of filaments in nonlinearly viscoelastic fluids. Phys. Fluids 21, 033102 (2009). 
99. Spagnolie, S. E., Liu, B. \& Powers, T. R. Locomotion of helical bodies in viscoelastic fluids: Enhanced swimming at large helical amplitudes. Phys. Rev. Lett. 111, 068101 (2013).

100. Man, Y. \& Lauga, E. Phase-separation models for swimming enhancement in complex fluids. Phys. Rev. E 92, 023004 (2015).

101. Liu, B., Powers, T. R. \& Breuer, K. S. Force-free swimming of a model helical flagellum in viscoelastic fluids. Proc. Natl. Acad. Sci. USA 108, 19516-19520 (2011).

102. Gagnon, D. A., Keim, N. C. \& Arratia, P. E. Undulatory swimming in shear-thinning fluids: experiments with caenorhabditis elegans. J. Fluid Mech. 758, R3 (2014).

103. Martinez, V. A., Schwarz-Linek, J., Reufer, M., Wilson, L. G., Morozov, A. N. \& Poon, W. C. K. Flagellated bacterial motility in polymer solutions. Proc. Natl. Acad. Sci. USA 111, 1777117776 (2014).

104. Zöttl, A. \& Yeomans, J. M. Enhanced bacterial swimming speeds in macromolecular polymer solutions. Nat. Phys. 15, 554-558 (2019).

105. McNamara, G. R. \& Zanetti, G. Use of the Boltzmann equation to simulate lattice-gas automata. Phys. Rev. Lett. 61, 23322335 (1988)

106. Dünweg, B. \& Ladd, A. J. C. Lattice Boltzmann simulations of soft matter systems. Adv. Polym. Sci. 221, 89-166 (2009).

107. Español, P. \& Warren, P. Statistical mechanics of dissipative particle dynamics. EPL 30, 191-196 (1995).

108. Kapral, R. Advances in Chemical Physics (John Wiley, UK, 2008).

109. Gompper, G., Ihle, T., Kroll, D. M. \& Winkler, R. G. Multiparticle collision dynamics: A particle-based mesoscale simulation approach to the hydrodynamics of complex fluids. $A d v$. Polym. Sci. 221, 1-87 (2009).

110. Ishikawa, T. \& Pedley, T. J. Coherent structures in monolayers of swimming particles. Phys. Rev. Lett. 100, 088103 (2008).

111. Mathijssen, A. J. T. M., Doostmohammadi, A., Yeomans, J. M. \& Shendruk, T. N. Hydrodynamics of micro-swimmers in films. J. Fluid Mech. 806, 35-70 (2016).

112. Singh, R., Ghose, S. \& Adhikari, R. Many-body microhydrodynamics of colloidal particles with active boundary layers. J. Stat. Mech. Theor. Exp. 2015, P06017 (2015).

113. Elgeti, J., Kaupp, U. B. \& Gompper, G. Hydrodynamics of sperm cells near surfaces. Biophys. J. 99, 1018-1026 (2010).

114. Watari, N. \& Larson, R. G. The hydrodynamics of a run-andtumble bacterium propelled by polymorphic helical flagella. Biophys. J. 98, 12-17 (2010).

115. Shum, H., Gaffney, E. A. \& Smith, D. J. Modelling bacterial behaviour close to a no-slip plane boundary: the influence of bacterial geometry. Proc. R. Soc. A 466, 1725-1748 (2010).

116. Pimponi, D., Chinappi, M., Gualtieri, P. \& Casciola, C. M. Hydrodynamics of flagellated microswimmers near free-slip interfaces. J. Fluid Mech. 789, 514-533 (2016).

117. Lighthill, M. J. On the squirming motion of nearly spherical deformable bodies through liquids at very small Reynolds numbers. Comm. Pure Appl. Math. 5, 109-118 (1952).

118. Blake, J. R. A spherical envelope approach to ciliary propulsion. J. Fluid Mech. 46, 199-208 (1971).

119. Ishikawa, T., Simmonds, M. P. \& Pedley, T. J. Hydrodynamic interaction of two swimming model micro-organisms. J. Fluid Mech. 568, 119-160 (2006).

120. Pedley, T. J. Spherical squirmers: models for swimming microorganisms. IMA J. Applied Mathematics 81, 488-521 (2016).

121. Llopis, I. \& Pagonabarraga, I. Hydrodynamic interactions in squirmer motion: Swimming with a neighbour and close to a wall. J. Non-Newtonian Fluid Mech. 165, 946-952 (2010).

122. Götze, I. O. \& Gompper, G. Mesoscale simulations of hydrodynamic squirmer interactions. Phys. Rev. E 82, 041921 (2010).

123. Evans, A. A., Ishikawa, T., Yamaguchi, T. \& Lauga, E. Orientational order in concentrated suspensions of spherical microswimmers. Phys. Fluids 23, 111702 (2011).

124. Alarcon, F. \& Pagonabarraga, I. Spontaneous aggregation and global polar ordering in squirmer suspensions. J. Mol. Liq. 185, 56-61 (2013).

125. Molina, J. J., Nakayama, Y. \& Yamamoto, R. Hydrodynamic interactions of self-propelled swimmers. Soft Matter 9, 49234936 (2013)

126. Yoshinaga, N. \& Liverpool, T. B. Hydrodynamic interactions in dense active suspensions: From polar order to dynamical clusters. Phys. Rev. E 96, 020603 (2017).

127. Ishimoto, K. \& Gaffney, E. A. Squirmer dynamics near a boundary. Phys. Rev. E 88, 062702 (2013).

128. Lintuvuori, J. S., Brown, A. T., Stratford, K. \& Marenduzzo, D. Hydrodynamic oscillations and variable swimming speed in squirmers close to repulsive walls. Soft Matter 12, 7959-7968 (2016).

129. Theers, M., Westphal, E., Gompper, G. \& Winkler, R. G. Modeling a spheroidal microswimmer and cooperative swimming in a narrow slit. Soft Matter 12, 7372-7385 (2016).

130. Theers, M., Westphal, E., Qi, K., Winkler, R. G. \& Gompper, G. Clustering of microswimmers: interplay of shape and hydrodynamics. Soft Matter 14, 8590-8603 (2018).

131. Keller, S. R. \& Wu, T. Y. A porous prolate-spheroidal model for ciliated micro-organisms. J. Fluid Mech. 80, 259-278 (1977).

132. Theers, M., Westphal, E., Gompper, G. \& Winkler, R. G. From local to hydrodynamic friction in Brownian motion: A multiparticle collision dynamics simulation study. Phys. Rev. E 93, 032604 (2016).

133. Nash, R. W., Adhikari, R., Tailleur, J. \& Cates, M. E. Run-andtumble particles with hydrodynamics: Sedimentation, trapping, and upstream swimming. Phys. Rev. Lett. 104, 258101 (2010).

134. Hernandez-Ortiz, J. P., Stoltz, C. G. \& Graham, M. D. Transport and collective dynamics in suspensions of confined swimming particles. Phys. Rev. Lett. 95, 204501 (2005).

135. de Graaf, J., Menke, H., Mathijssen, A. J. T. M., Fabritius, M., Holm, C. \& Shendruk, T. N. Lattice Boltzmann hydrodynamics of anisotropic active matter. J. Chem. Phys. 144, 134106 (2016).

136. Menzel, A. M., Saha, A., Hoell, C. \& Löwen, H. Dynamical density functional theory for microswimmers. J. Chem. Phys. 144, 024115 (2016).

137. Lighthill, J. Flagellar hydrodynamics. SIAM Rev. 18, 161-230 (1976).

138. Saggiorato, G., Alvarez, L., Jikeli, J. F., Kaupp, U. B., Gompper, G. \& Elgeti, J. Human sperm steer with second harmonics of the flagellar beat. Nat. Commun. 8, 1415 (2017).

139. Shum, H. \& Gaffney, E. A. Hydrodynamic analysis of flagellated bacteria swimming near one and between two no-slip plane boundaries. Phys. Rev. E 91, 033012 (2015).

140. Lauga, E. Bacterial hydrodynamics. Annu. Rev. Fluid Mech. 48, 105-130 (2016).

141. Rode, S., Elgeti, J. \& Gompper, G. Sperm motility in modulated microchannels. New J. Phys. 21, 013016 (2019).

142. Reigh, S. Y., Winkler, R. G. \& Gompper, G. Synchronization and bundling of anchored bacterial flagella. Soft Matter $\mathbf{8}$, 4363-4372 (2012).

143. Reichert, M. \& Stark, H. Synchronization of rotating helices by hydrodynamic interactions. Eur. Phys. J. E 17, 493-500 (2005).

144. Vogel, R. \& Stark, H. Motor-driven bacterial flagella and buckling instabilities. Eur. Phys. J. E 35, 15 (2012).

145. Janssen, P. J. A. \& Graham, M. D. Coexistence of tight and loose bundled states in a model of bacterial flagellar dynamics. Phys. Rev. E 84, 011910 (2011).

146. Hu, J., Wysocki, A., Winkler, R. G. \& Gompper, G. Physical sensing of surface properties by microswimmers - directing bacterial motion via wall slip. Sci. Rep. 5, 9586 (2015).

147. Lemelle, L., Palierne, J.-F., Chatre, E., Vaillant, C. \& Place, C. Curvature reversal of the circular motion of swimming bacteria probes for slip at solid/liquid interfaces. Soft Matter 9, 9759-9762 (2013).

148. Lauga, E., DiLuzio, W. R., Whitesides, G. M. \& Stone, H. A. 
Swimming in circles: Motion of bacteria near solid boundaries. Biophys. J. 90, 400-412 (2006).

149. Di Leonardo, R., Dell Arciprete, D., Angelani, L. \& Iebba, V. Swimming with an image. Phys. Rev. Lett. 106, 038101 (2011).

150. Dombrowski, C., Cisneros, L., Chatkaew, S., Goldstein, R. E. \& Kessler, J. O. Self-concentration and large-scale coherence in bacterial dynamics. Phys. Rev. Lett. 93, 098103 (2004).

151. Bialké, J., Speck, T. \& Löwen, H. Crystallization in a dense suspension of self-propelled particles. Phys. Rev. Lett. 108, 168301 (2012).

152. Matas-Navarro, R., Golestanian, R., Liverpool, T. B. \& Fielding, S. M. Hydrodynamic suppression of phase separation in active suspensions. Phys. Rev. E 90, 032304 (2014).

153. Gaspard, P. \& Kapral, R. Thermodynamics and statistical mechanics of chemically powered synthetic nanomotors. Advances in Physics: X 4, 1602480 (2019).

154. Bayati, P., Popescu, M. N., Uspal, W. E., Dietrich, S. \& Najafi, A. Dynamics near planar walls for various model self-phoretic particles. Soft Matter 15, 5644-5672 (2019).

155. Rückner, G. \& Kapral, R. Chemically powered nanodimers. Phys. Rev. Lett. 98, 150603 (2007).

156. Yang, M. \& Ripoll, M. Simulations of thermophoretic nanoswimmers. Phys. Rev. E 84, 061401 (2011).

157. Saha, S., Golestanian, R. \& Ramaswamy, S. Clusters, asters, and collective oscillations in chemotactic colloids. Phys. Rev. E 89, 062316 (2014).

158. Michelin, S. \& Lauga, E. Phoretic self-propulsion at finite Péclet numbers. J. Fluid Mech. 747, 572 (2014).

159. Liebchen, B., Marenduzzo, D., Pagonabarraga, I. \& Cates, M. E. Clustering and pattern formation in chemorepulsive active colloids. Phys. Rev. Lett. 115, 258301 (2015).

160. Stark, H. Artificial chemotaxis of self-phoretic active colloids: Collective behavior. Acc. Chem. Res. 51, 2681-2688 (2018).

161. Moran, J. L. \& Posner, J. D. Phoretic self-propulsion. Annu. Rev. Fluid Mech. 49, 511-540 (2017).

162. Howse, J. R., Jones, R. A. L., Ryan, A. J., Gough, T., Vafabakhsh, R. \& Golestanian, R. Self-motile colloidal particles: From directed propulsion to random walk. Phys. Rev. Lett. 99, 048102 (2007).

163. Uspal, W. E., Popescu, M. N., Dietrich, S. \& Tasinkevych, M. Self-propulsion of a catalytically active particle near a planar wall: from reflection to sliding and hovering. Soft Matter 11, 434 (2015)

164. Ishimoto, K. \& Gaffney, E. A. Fluid flow and sperm guidance: a simulation study of hydrodynamic sperm rheotaxis. J. Roy. Soc. Interface 12, 20150172 (2015).

165. Koh, J. B. Y., Shen, X. \& Marcos. Theoretical modeling in microscale locomotion. Microfluidics and Nanofluidics 20, 98 (2016).

166. Uspal, W. E., Popescu, M. N., Dietrich, S. \& Tasinkevych, M. Rheotaxis of spherical active particles near a planar wall. Soft Matter 11, 6613-6632 (2015).

167. Mathijssen, A., Figueroa-Morales, N., Junot, G., Clement, E., Lindner, A. \& Zöttl, A. Oscillatory surface rheotaxis of swimming E. coli bacteria. Nat. Commun. 20, 3434 (2019).

168. Friedrich, B. M. \& Jülicher, F. Chemotaxis of sperm cells. Proc. Natl. Acad. Sci. USA 104, 13256 (2007).

169. Tu, Y. Quantitative modeling of bacterial chemotaxis: Signal amplification and accurate adaptation. Annu. Rev. Biophys. 42, 337-359 (2013).

170. Camley, B. A., Zimmermann, J., Levine, H. \& Rappel, W.-J. Emergent collective chemotaxis without single-cell gradient sensing. Phys. Rev. Lett. 116, 098101 (2016).

171. ten Hagen, B., Kümmel, F., Wittkowski, R., Takagi, D., Löwen, H. \& Bechinger, C. Gravitaxis of asymmetric self-propelled colloidal particles. Nat. Commun. 5, 4829 (2014).

172. Kuhr, J.-T., Blaschke, J., Rühle, F. \& Stark, H. Collective sedimentation of squirmers under gravity. Soft Matter 13, 7548-7555 (2017).

173. Cohen, J. A. \& Golestanian, R. Emergent cometlike swarming of optically driven thermally active colloids. Phys. Rev. Lett. 112, 068302 (2014).

174. Martin, P. C., Parodi, O. \& Pershan, P. S. Unified hydrodynamic theory for crystals, liquid crystals, and normal fluids. Phys. Rev. A 6, 2401-2420 (1972).

175. Prost, J., Jülicher, F. \& Joanny, J.-F. Active gel physics. Nat. Phys. 11, 111-117 (2015).

176. Jülicher, F., Grill, S. W. \& Salbreux, G. Hydrodynamic theory of active matter. Rep. Prog. Phys. 81, 076601 (2018).

177. Carenza, L. N., Gonnella, G., Lamura, A., Negro, G. \& Tiribocchi, A. Lattice boltzmann methods and active fluids. The European Physical Journal E 42, 81 (2019).

178. Aditi Simha, R. \& Ramaswamy, S. Hydrodynamic fluctuations and instabilities in ordered suspensions of self-propelled particles. Phys. Rev. Lett. 89, 058101 (2002).

179. Hatwalne, Y., Ramaswamy, S., Rao, M. \& Simha, R. A. Rheology of active-particle suspensions. Phys. Rev. Lett. 92, 118101 (2004).

180. Kruse, K., Joanny, J. F., Jülicher, F., Prost, J. \& Sekimoto, K. Asters, vortices, and rotating spirals in active gels of polar filaments. Phys. Rev. Lett. 92, 078101 (2004).

181. Giomi, L., Marchetti, M. C. \& Liverpool, T. B. Complex spontaneous flows and concentration banding in active polar films. Phys. Rev. Lett. 101, 198101 (2008).

182. Baskaran, A. \& Marchetti, M. C. Statistical mechanics and hydrodynamics of bacterial suspensions. Proc. Natl. Acad. Sci. USA 106, 15567-15572 (2009).

183. Linkmann, M., Marchetti, M. C., Boffetta, G. \& Eckhardt, B. Condensate formation and multiscale dynamics in two-dimensional active suspensions. arXiv e-prints arXiv:1905.06267 (2019).

184. Marenduzzo, D., Orlandini, E., Cates, M. E. \& Yeomans, J. M. Steady-state hydrodynamic instabilities of active liquid crystals: Hybrid lattice Boltzmann simulations. Phys. Rev. E 76, 031921 (2007).

185. Marenduzzo, D., Orlandini, E. \& Yeomans, J. M. Hydrodynamics and rheology of active liquid crystals: A numerical investigation. Phys. Rev. Lett. 98, 118102 (2007).

186. Giomi, L., Mahadevan, L., Chakraborty, B. \& Hagan, M. F. Excitable patterns in active nematics. Phys. Rev. Lett. 106, 218101 (2011).

187. Doostmohammadi, A., Ignes-Mullol, J., Yeomans, J. M. \& Sagues, F. Active nematics. Nat. Commun. 9, 3246 (2018).

188. Dunkel, J., Heidenreich, S., Bär, M. \& Goldstein, R. E. Minimal continuum theories of structure formation in dense active fluids. New J. Phys. 15, 045016 (2013).

189. Slomka, J. \& Dunkel, J. Generalized navier-stokes equations for active suspensions. Eur. Phys. J. Spec. Top. 224, 1349-1358 (2015).

190. Dunkel, J., Heidenreich, S., Drescher, K., Wensink, H. H., Bär, M. \& Goldstein, R. E. Fluid dynamics of bacterial turbulence. Phys. Rev. Lett. 110, 228102 (2013).

191. Wensink, H. H., Dunkel, J., Heidenreich, S., Drescher, K., Goldstein, R. E., Löwen, H. \& Yeomans, J. M. Meso-scale turbulence in living fluids. Proc. Natl. Acad. Sci. USA 109, 14308-14313 (2012).

192. Slomka, J. \& Dunkel, J. Geometry-dependent viscosity reduction in sheared active fluids. Phys. Rev. Fluids 2, 043102 (2017).

193. Slomka, J. \& Dunkel, J. Spontaneous mirror-symmetry breaking induces inverse energy cascade in $3 \mathrm{~d}$ active fluids. Proc. Natl. Acad. Sci. USA 114, 2119-2124 (2017).

194. Tiribocchi, A., Wittkowski, R., Marenduzzo, D. \& Cates, M. E. Active model h: Scalar active matter in a momentumconserving fluid. Phys. Rev. Lett. 115, 188302 (2015).

195. Cates, M. E. Active Field Theories. arXiv e-prints arXiv:1904.01330 (2019).

196. Mogilner, A. \& Oster, G. Cell motility driven by actin polymerization. Biophys. J. 71, 3030-3045 (1996).

197. Dogterom, M., Kerssemakers, J. W., Romet-Lemonne, G. \& Janson, M. E. Force generation by dynamic microtubules. 
Curr. Opin. Cell Biol. 17, 67-74 (2005).

198. Mogilner, A. Mathematics of cell motility: have we got its number? J. Math. Biol. 58, 105 (2008).

199. Erlenkämper, C. \& Kruse, K. Uncorrelated changes of subunit stability can generate length-dependent disassembly of treadmilling filaments. Phys. Biol. 6, 046016 (2009).

200. Howard, J. Mechanics of motor proteins and the cytoskeleton (Sinauer Associates, Sunderland, 2001).

201. Nielsen, S. O., Bulo, R. E., Moore, P. B. \& Ensing, B. Recent progress in adaptive multiscale molecular dynamics simulations of soft matter. Phys. Chem. Chem. Phys. 12, 1240112414 (2010).

202. Ekimoto, T. \& Ikeguchi, M. Multiscale molecular dynamics simulations of rotary motor proteins. Biophys. Rev. 10, 605615 (2018)

203. Kolomeisky, A. B. \& Fisher, M. E. Molecular motors: A theorist's perspective. Annu. Rev. Phys. Chem. 58, 675-695 (2007).

204. Chowdhury, D. Stochastic mechano-chemical kinetics of molecular motors: A multidisciplinary enterprise from a physicist's perspective. Phys. Rep. 529, 1-197 (2013).

205. Klumpp, S. \& Lipowsky, R. Cooperative cargo transport by several molecular motors. Proc. Natl. Acad. Sci. USA 102, 17284-17289 (2005).

206. Appert-Rolland, C., Ebbinghaus, M. \& Santen, L. Intracellular transport driven by cytoskeletal motors: General mechanisms and defects. Phys. Rep. 593, 1-59 (2015).

207. Bausch, A. R. \& Kroy, K. A bottom-up approach to cell mechanics. Nat. Phys. 2, 231-238 (2006).

208. Huber, F., Schnauß, J., Rönicke, S., Rauch, P., Müller, K., Fütterer, C. \& Käs, J. Emergent complexity of the cytoskeleton: from single filaments to tissue. Adv. Phys. 62, 1-112 (2013).

209. Broedersz, C. P. \& MacKintosh, F. C. Modeling semiflexible polymer networks. Rev. Mod. Phys. 86, 995-1036 (2014).

210. Nedelec, F. \& Foethke, D. Collective langevin dynamics of flexible cytoskeletal fibers. New J. Phys. 9, 427-427 (2007).

211. Mohapatra, L., Goode, B. L., Jelenkovic, P., Phillips, R. \& Kondev, J. Design principles of length control of cytoskeletal structures. Annu. Rev. Biophys. 45, 85-116 (2016).

212. Mogilner, A. \& Craig, E. Towards a quantitative understanding of mitotic spindle assembly and mechanics. J. Cell Sci. 123, 3435-3445 (2010).

213. Pavin, N. \& Tolić, I. M. Self-organization and forces in the mitotic spindle. Annu. Rev. Biophys. 45, 279-298 (2016).

214. Broedersz, C. P. \& MacKintosh, F. C. Molecular motors stiffen non-affine semiflexible polymer networks. Soft Matter 7, 3186-3191 (2011).

215. Ronceray, P., Broedersz, C. P. \& Lenz, M. Fiber networks amplify active stress. Proc. Natl. Acad. Sci. USA 113, 28272832 (2016).

216. Jülicher, F., Kruse, K., Prost, J. \& Joanny, J.-F. Active behavior of the cytoskeleton. Phys. Rep. 449, 3-28 (2007).

217. Joanny, J. F. \& Prost, J. Active gels as a description of the actin-myosin cytoskeleton. HFSP J. 3, 94-104 (2009).

218. Jilkine, A. \& Edelstein-Keshet, L. A comparison of mathematical models for polarization of single eukaryotic cells in response to guided cues. PLoS Comput. Biol. 7, e1001121 (2011).

219. Holmes, W. R. \& Edelstein-Keshet, L. A comparison of computational models for eukaryotic cell shape and motility. PLOS Comput. Biol. 8, e1002793 (2012).

220. Danuser, G., Allard, J. \& Mogilner, A. Mathematical modeling of eukaryotic cell migration: Insights beyond experiments. Annu. Rev. Cell Dev. Biol. 29, 501-528 (2013).

221. te Boekhorst, V., Preziosi, L. \& Friedl, P. Plasticity of cell migration in vivo and in silico. Annu. Rev. Cell Dev. Biol. 32, 491-526 (2016).

222. Doubrovinski, K. \& Kruse, K. Cell motility resulting from spontaneous polymerization waves. Phys. Rev. Lett. 107, 258103 (2011).

223. Wolgemuth, C. W., Stajic, J. \& Mogilner, A. Redundant mechanisms for stable cell locomotion revealed by minimal models.
Biophys. J. 101, 545-553 (2011).

224. Ziebert, F., Swaminathan, S. \& Aranson, I. S. Model for selfpolarization and motility of keratocyte fragments. J. Royal Soc. Interface 9, 1084-1092 (2012).

225. Ziebert, F. \& Aranson, I. S. Computational approaches to substrate-based cell motility. npj Comput. Mater. 2, 16019 (2016).

226. Linsmeier, I., Banerjee, S., Oakes, P. W., Jung, W., Kim, T. \& Murrell, M. P. Disordered actomyosin networks are sufficient to produce cooperative and telescopic contractility. Nat. Commun. 7, 12615 (2016).

227. Singer-Loginova, I. \& Singer, H. M. The phase field technique for modeling multiphase materials. Rep. Prog. Phys. 71, 106501 (2008).

228. Nonomura, M. Study on multicellular systems using a phase field model. PLOS ONE 7, 1-9 (2012).

229. Camley, B. A., Zhang, Y., Zhao, Y., Li, B., Ben-Jacob, E., Levine, H. \& Rappel, W.-J. Polarity mechanisms such as contact inhibition of locomotion regulate persistent rotational motion of mammalian cells on micropatterns. Proc. Natl. Acad. Sci. USA 111, 14770-14775 (2014).

230. Najem, S. \& Grant, M. Phase-field model for collective cell migration. Phys. Rev. E 93, 052405 (2016).

231. Camley, B. A. \& Rappel, W.-J. Physical models of collective cell motility: from cell to tissue. J. Phys. D 50, 113002 (2017).

232. Mueller, R., Yeomans, J. M. \& Doostmohammadi, A. Emergence of active nematic behavior in monolayers of isotropic cells. Phys. Rev. Lett. 122, 048004 (2019).

233. Wenzel, D., Praetorius, S. \& Voigt, A. Topological and geometrical quantities in active cellular structures. J. Chem. Phys. 150, 164108 (2019).

234. Abaurrea-Velasco, C., Ghahnaviyeh, S. D., Pishkenari, H. N., Auth, T. \& Gompper, G. Complex self-propelled rings: a minimal model for cell motility. Soft Matter 13, 5865-5876 (2017).

235. Abaurrea-Velasco, C., Auth, T. \& Gompper, G. Self-organized motility of vesicles with internal active filaments. arXiv eprints arXiv:1812.09932 (2019).

236. Preziosi, L., Ambrosi, D. \& Verdier, C. An elasto-visco-plastic model of cell aggregates. J. Theor. Biol. 262, 35-47 (2010).

237. Rodriguez, E. K., Hoger, A. \& McCulloch, A. D. Stressdependent finite growth in soft elastic tissues. J. Biomech. 27, 455-467 (1994).

238. Drasdo, D. \& Höhme, S. A single-cell-based model of tumor growthin vitro: monolayers and spheroids. Phys. Biol. 2, 133-147 (2005).

239. Basan, M., Prost, J., Joanny, J.-F. \& Elgeti, J. Dissipative particle dynamics simulations for biological tissues: rheology and competition. Phys. Biol. 8, 026014 (2011).

240. Malmi-Kakkada, A. N., Li, X., Samanta, H. S., Sinha, S. \& Thirumalai, D. Cell growth rate dictates the onset of glass to fluidlike transition and long time superdiffusion in an evolving cell colony. Phys. Rev. X 8, 021025 (2018).

241. Matoz-Fernandez, D. A., Martens, K., Sknepnek, R., Barrat, J. L. \& Henkes, S. Cell division and death inhibit glassy behaviour of confluent tissues. Soft Matter 13, 3205-3212 (2017).

242. Graner, F. \& Glazier, J. A. Simulation of biological cell sorting using a two-dimensional extended potts model. Phys. Rev. Lett. 69, 2013-2016 (1992).

243. Glazier, J. A. \& Graner, F. Simulation of the differential adhesion driven rearrangement of biological cells. Phys. Rev. E 47, 2128-2154 (1993).

244. Chen, N., Glazier, J. A., Izaguirre, J. A. \& Alber, M. S. A parallel implementation of the cellular potts model for simulation of cell-based morphogenesis. Comput. Phys. Commun. 176, 670681 (2007).

245. Maree, A. F. M. \& Hogeweg, P. How amoeboids self-organize into a fruiting body: Multicellular coordination in dictyostelium discoideum. Proc. Natl. Acad. Sci. USA 98, 38793883 (2001). 
246. Merks, R. M. H., Perryn, E. D., Shirinifard, A. \& Glazier, J. A. Contact-inhibited chemotaxis in de novo and sprouting bloodvessel growth. PLoS Comp. Biol. 4, e1000163 (2008).

247. Maree, A. F. M., Jilkine, A., Dawes, A., Grieneisen, V. A. \& Edelstein-Keshet, L. Polarization and movement of keratocytes: A multiscale modelling approach. Bull. Math. Biol. 68, 1169-1211 (2006).

248. Albert, P. J. \& Schwarz, U. S. Dynamics of cell shape and forces on micropatterned substrates predicted by a cellular potts model. Biophys. J. 106, 2340-2352 (2014).

249. Segerer, F. J., Thüroff, F., Piera Alberola, A., Frey, E. \& Rädler, J. O. Emergence and persistence of collective cell migration on small circular micropatterns. Phys. Rev. Lett. 114, 228102 (2015).

250. Hufnagel, L., Teleman, A. A., Rouault, H., Cohen, S. M. \& Shraiman, B. I. On the mechanism of wing size determination in fly development. Proc. Natl. Acad. Sci. USA 104, 3835-3840 (2007).

251. Fletcher, A. G., Osterfield, M., Baker, R. E. \& Shvartsman, S. Y. Vertex models of epithelial morphogenesis. Biophys. J. 106, 2291-2304 (2014).

252. Sussman, D. M., Schwarz, J. M., Marchetti, M. C. \& Manning, M. L. Soft yet sharp interfaces in a vertex model of confluent tissue. Phys. Rev. Lett. 120, 058001 (2018).

253. Barton, D. L., Henkes, S., Weijer, C. J. \& Sknepnek, R. Active vertex model for cell-resolution description of epithelial tissue mechanics. PLOS Comput. Biol. 13, e1005569 (2017).

254. Oswald, L., Grosser, S., Smith, D. M. \& Käs, J. A. Jamming transitions in cancer. J. Phys. D 50, 483001 (2017).

255. Chiang, M. \& Marenduzzo, D. Glass transitions in the cellular potts model. Europhys. Lett. 116, 28009 (2016).

256. Bi, D., Yang, X., Marchetti, M. C. \& Manning, M. L. Motilitydriven glass and jamming transitions in biological tissues. Phys. Rev. X 6, 021011 (2016).

257. Fung, Y.-C. Biomechanics: Mechanical Properties of Living Tissues, 2nd ed. (Springer New York, 2010).

258. Dunlop, J. W. C., Fischer, F. D., Gamsjäger, E. \& Fratzl, P. A theoretical model for tissue growth in confined geometries. J. Mech. Phys. Solids 58, 1073-1087 (2010).

259. Ambrosi, D., Preziosi, L. \& Vitale, G. The interplay between stress and growth in solid tumors. Mech. Res. Commun. 42, 87-91 (2012).

260. Shraiman, B. I. Mechanical feedback as a possible regulator of tissue growth. Proc. Natl. Acad. Sci. USA 102, 3318-3323 (2005).

261. Byrne, H. \& Preziosi, L. Modelling solid tumour growth using the theory of mixtures. Math. Med. Biol. 20, 341-366 (2003).

262. Tracqui, P. Biophysical models of tumour growth. Rep. Prog. Phys. 72, 056701 (2009).

263. Rieger, H. \& Welter, M. Integrative models of vascular remodeling during tumor growth. WIREs Syst. Biol. Med. 7, 113-129 (2015).

264. Fredrich, T., Rieger, H., Chignola, R. \& Milotti, E. Fine-grained simulations of the microenvironment of vascularized tumours. Sci. Rep. 9, 11698 (2019).

265. Romanczuk, P., Couzin, I. D. \& Schimansky-Geier, L. Collective motion due to individual escape and pursuit response. Phys. Rev. Lett. 102, 010602 (2009).

266. Simpson, S. J., Sword, G. A., Lorch, P. D. \& Couzin, I. D. Cannibal crickets on a forced march for protein and salt. Proc. Natl. Acad. Sci. USA 103, 4152-4156 (2006).

267. Agudo-Canalejo, J. \& Golestanian, R. Active phase separation in mixtures of chemically interacting particles. Phys. Rev. Lett. 123, 018101 (2019).

268. Pearce, D. J. G., Miller, A. M., Rowlands, G. \& Turner, M. S. Role of projection in the control of bird flocks. Proc. Natl. Acad. Sci. USA 111, 10422-10426 (2014).

269. Lavergne, F. A., Wendehenne, H., Bäuerle, T. \& Bechinger, C. Group formation and cohesion of active particles with visual perception-dependent motility. Science 364, 70-74 (2019).

270. Ballerini, M., Cabibbo, N., Candelier, R., Cavagna, A., Cisbani,
E., Giardina, I., Lecomte, V., Orlandi, A., Parisi, G., Procaccini, A., Viale, M. \& Zdravkovic, V. Interaction ruling animal collective behavior depends on topological rather than metric distance: Evidence from a field study. Proc. Natl. Acad. Sci. USA 105, 1232-1237 (2008).

271. Bajec, I. L. \& Heppner, F. H. Organized flight in birds. Animal Behav. 78, 777-789 (2009).

272. Mijalkov, M., McDaniel, A., Wehr, J. \& Volpe, G. Engineering sensorial delay to control phototaxis and emergent collective behaviors. Phys. Rev. X 6, 011008 (2016).

273. Charlesworth, H. J. \& Turner, M. S. Intrinsically motivated collective motion. Proc. Natl. Acad. Sci. USA 116, 1536215367 (2019).

274. Khadka, U., Holubec, V., Yang, H. \& Cichos, F. Active particles bound by information flows. Nat. Commun. 9, 3864 (2018).

275. Mann, R. P. \& Garnett, R. The entropic basis of collective behaviour. J. R. Soc. Interface 12, 20150037 (2015)

276. Ward, A. J. W., Sumpter, D. J. T., Couzin, I. D., Hart, P. J. B. \& Krause, J. Quorum decision-making facilitates information transfer in fish shoals. Proc. Natl. Acad. Sci. USA 105, 69486953 (2008).

277. Abaurrea Velasco, C., Abkenar, M., Gompper, G. \& Auth, T. Collective behavior of self-propelled rods with quorum sensing. Phys. Rev. E 98, 022605 (2018).

278. Castellano, C., Fortunato, S. \& Loreto, V. Statistical physics of social dynamics. Rev. Mod. Phys. 81, 591-646 (2009).

279. King, A. J., Douglas, C. M., Huchard, E., Isaac, N. J. \& Cowlishaw, G. Dominance and affiliation mediate despotism in a social primate. Curr. Biol. 18, 1833-1838 (2008).

280. Couzin, I. D., Krause, J., Franks, N. R. \& Levin, S. A. Effective leadership and decision-making in animal groups on the move. Nature 433, 513-516 (2005).

281. Freeman, R. \& Biro, D. Modelling group navigation: Dominance and democracy in homing pigeons. J. Navigation 62, 33-40 (2009).

282. Bäuerle, T., Fischer, A., Speck, T. \& Bechinger, C. Selforganization of active particles by quorum sensing rules. Nat. Commun. 9, 3232 (2018).

283. Moussaïd, M., Helbing, D. \& Theraulaz, G. How simple rules determine pedestrian behavior and crowd disasters. Proc. Natl. Acad. Sci. USA 108, 6884-6888 (2011).

284. Faria, J. J., Dyer, J. R., Tosh, C. R. \& Krause, J. Leadership and social information use in human crowds. Animal Behav. 79, 895-901 (2010).

285. Bain, N. \& Bartolo, D. Dynamic response and hydrodynamics of polarized crowds. Science 363, 46-49 (2019).

286. Kim, M.-C., Silberberg, Y. R., Abeyaratne, R., Kamm, R. D. \& Asada, H. H. Computational modeling of three-dimensional ecm-rigidity sensing to guide directed cell migration. Proc. Natl. Acad. Sci. USA 115, E390-E399 (2018).

287. Paluch, E. K. \& Raz, E. The role and regulation of blebs in cell migration. Curr. Opin. Cell Biol. 25, 582-590 (2013).

288. Tozluoglu, M., Tournier, A. L., Jenkins, R. P., Hooper, S., Bates, P. A. \& Sahai, E. Matrix geometry determines optimal cancer cell migration strategy and modulates response to interventions. Nat. Cell Biol. 15, 751 (2013).

289. Besser, A. \& Schwarz, U. S. Coupling biochemistry and mechanics in cell adhesion: a model for inhomogeneous stress fiber contraction. New J. Phys. 9, 425-425 (2007).

290. Nishikawa, M., Naganathan, S. R., Jülicher, F. \& Grill, S. W. Controlling contractile instabilities in the actomyosin cortex. eLife 6, e19595 (2017).

291. Gross, P., Kumar, K. V., Goehring, N. W., Bois, J. S., Hoege, C., Jülicher, F. \& Grill, S. W. Guiding self-organized pattern formation in cell polarity establishment. Nat. Phys. 15, 293300 (2019). 


\begin{tabular}{|c|c|c|}
\hline Package & Scope \& Features & URL \\
\hline CytoSim & $\begin{array}{l}\text { Simulation of an ensemble of semi-flexible cytoskeletal } \\
\text { filaments and associated proteins found inside of eukaryotic cells }\end{array}$ & http://www.cytosim.org/ \\
\hline AFINES & $\begin{array}{l}\text { coarse-grained simulation of cytoskeleton, consisting of } \\
\text { semiflexible filaments, crosslinkers, and protein motors }\end{array}$ & $\begin{array}{c}\text { http://dinner-group.uchicago.edu } \\
\text { /downloads/ }\end{array}$ \\
\hline MEDYAN & $\begin{array}{c}\text { mechanochemical simulation of filamentous systems, } \\
\text { such as actomyosin network }\end{array}$ & http://www.medyan.org/ \\
\hline SAMOS & actomyosin and tissue mechanics simulation & $\begin{array}{l}\text { https://github.com/sknepneklab } \\
\text { /SAMoS/ }\end{array}$ \\
\hline CHASTE & a multiscale computational framework for modeling cell populations & https://www.cs.ox.ac.uk/chaste/ \\
\hline COMPUCELL & $\begin{array}{l}\text { multi-scale modeling of multi-cellular systems, such as bacterial } \\
\text { colonies, cancer, and immune systems, based on the cellular Potts model }\end{array}$ & http://www.compucell3d.org/ \\
\hline cellGPU & simulation of Voronoi and vertex models of cells & $\begin{array}{l}\text { https://gitlab.com/dmsussman } \\
\text { /cellGPU/ }\end{array}$ \\
\hline
\end{tabular}

\section{Simulation packages for hydrodynamics and self-propelled particles}

\begin{tabular}{ccc|} 
Package & \multicolumn{1}{c}{ Scope \& Features } & \multicolumn{1}{c}{ URL } \\
$\begin{array}{c}\text { palabos, OpenLB, } \\
\text { Musubi }\end{array}$ & $\begin{array}{c}\text { computational fluid dynamics solvers } \\
\text { based on the lattice Boltzmann approach }\end{array}$ & $\begin{array}{c}\text { http://www.palabos.org/ } \\
\text { https://www.openlb.net/overview/ } \\
\text { https://geb.sts.nt.uni-siegen.de } \\
\text { /doxy/musubi/index.html/ }\end{array}$ \\
\hline ESPResSo & $\begin{array}{c}\text { molecular dynamics many-particle simulation of } \\
\text { coarse-grained atomistic or bead-spring models }\end{array}$ & http://espressomd.org/wordpress/ \\
\hline LAMMPS & $\begin{array}{c}\text { a massively parallel molecular dynamics code } \\
\text { for large-scale soft particle simulation }\end{array}$ & https://lammps.sandia.gov/ \\
\hline KAPSEL & $\begin{array}{c}\text { simulation of solid particles dispersed in complex fluids } \\
\text { based on a Direct Numerical Simulation method (DNS) }\end{array}$ & https://kapsel-dns.com/ \\
\hline
\end{tabular}

TABLE S1: (top) Computational packages for modeling biological active matter. (bottom) A few particle-based software packages with the option of simulating active particle systems. 